\title{
Polarimetric Calibration of the Ku-Band Advanced Polarimetric Radar Interferometer
}

\section{Journal Article}

Author(s):

Baffelli, Simone; Frey, Othmar (1D; Werner, Charles; Hajnsek, Irena

Publication date:

2018-04

Permanent link:

https://doi.org/10.3929/ethz-b-000223398

Rights / license:

In Copyright - Non-Commercial Use Permitted

Originally published in:

IEEE Transactions on Geoscience and Remote Sensing 56(4), https://doi.org/10.1109/tgrs.2017.2778049 
Author's post-print (ie final draft post-refereeing), accepted for publication/published in the IEEE Transactions on Geoscience And Remote Sensing (2017-12-19).

(c) 2017 IEEE. Personal use of this material is permitted. Permission from IEEE must be obtained for all other users, including reprinting/ republishing this material for advertising or promotional purposes, creating new collective works for resale or redistribution to servers or lists, or reuse of any copyrighted components of this work in other works.

doi:10.1109/TGRS.2017.2778049, url:http://ieeexplore.ieee.org/document/8226855/ 


\title{
Polarimetric Calibration of the Ku Band Advanced Polarimetric Radar Interferometer (KAPRI)
}

\author{
Simone Baffelli, Student Member, IEEE, Othmar Frey, Senior Member, IEEE, Charles Werner, Senior \\ Member, IEEE, and Irena Hajnsek, Fellow, IEEE
}

\begin{abstract}
Differential interferometry using groundbased radar systems permits to monitor displacements in natural terrain with high flexibility in location, time of acquisition and revisit time. In combination with polarimetric imaging, discrimination of different scattering mechanisms present in a resolution cell can be obtained simultaneously with the estimation of surface displacement. In this paper we present the pre-processing steps and the calibration procedure required to produce high-quality calibrated polarimetric single-look complex imagery with KAPRI, a new portable Ku-band polarimetric radar interferometer. The processing of KAPRI data into SLC images is addressed, including the correction of beam squint and of azimuthal phase variations. A polarimetric calibration model adapted to the acquisition mode is presented and used to produce calibrated polarimetric covariance matrix data. The methods are validated by means of a scene containing five trihedral corner reflectors. Data pre-processing is assessed by analyzing the oversampled response of a corner reflector and the polarimetric calibration quality is verified by computing polarimetric signatures and residual calibration parameters.
\end{abstract}

Index Terms-Ground based radar, polarimetric radar, polarimetric calibration

\section{INTRODUCTION}

D IFFERENTIAL radar interferometry [1]-[4] is widely used to monitor and study changes in the

Manuscript received June 12, 2017; revised September 14, 2017; accepted October 29, 2017. (Corresponding author: Simone Baffelli)

$\mathrm{S}$. Baffelli is with the Chair of Earth Observation and Remote Sensing, Institute of Environmental Engineering, ETH Zurich, 8093 Zurich, Switzerland (e-mail: bafsimon@ethz.ch).

O. Frey is with the Chair of Earth Observation and Remote Sensing, Institute of Environmental Engineering, ETH Zurich, 8093 Zurich, Switzerland, and also with GAMMA Remote Sensing AG, 3073 Gümligen, Switzerland (e-mail: otfrey@ethz.ch/frey@gamma-rs.ch).

C. Werner is with GAMMA Remote Sensing AG, 3073 Güumligen, Switzerland (e-mail: cw@gamma-rs.ch).

I. Hajnsek is with the Chair of Earth Observation and Remote Sensing, Institute of Environmental Engineering, ETH Zurich, 8093 Zurich, Switzerland, and also with the Microwaves and Radar Institute, German Aerospace Center, 82234 Wessling, Germany (e-mail: hajnsek@ifu.baug.ethz.ch).

Digital Object Identifier 10.1109/TGRS.2017.2778049 natural and built environment. The ability to measure the line-of-sight component of movements over large areas makes it suitable for many applications. Some examples are the estimation of subsidence rate associated with temporal changes in the water table of aquifers, oil and gas extraction, deep mining and tunnel excavation [5][7], the monitoring of inflation/deflation connected to volcanic activity [8], the mapping of ice sheet and glacier motion [9], [10], the observation of instable slopes in rugged natural terrain and open pit mines [11], [12] and the measurement of seismic displacements [13], [14].

Fully polarimetric radar data provides additional information on the scattering mechanism within each resolution cell, which is employed for classification of the surface cover [15], [16], to extract geophysical parameters such as moisture content [17], to estimate the orientation of the vegetation canopy [18] or the height of fresh snow [19].

The availability of polarimetric information in addition to the interferometric time series allows to combine the scattering matrix and the interferometric coherence to better characterize the natural processes observed with the radar: a salient example being coherence optimization, where the scattering mechanism providing the best coherence and thus the least noisy phase measurement is selected [20], [21].

Spaceborne SAR systems, such as ERS-1 and 2, Envisat/ASAR, TerraSAR-X, Sentinel 1-A/1-B, Radarsat1 and 2 and ALOS, ALOS 2, Cosmo SkyMed, have been an important data source to build up interferometric time series for displacement measurements sometimes spanning over many years. These platforms are convenient in that they offer a large coverage in a single pass. However, the revisit time of these system is limited to few hours at best. In many cases, to better understand the dynamics of natural processes and for real-time surveillance and alarming, a denser temporal sampling over longer time spans and other observation geometries are desired than the ones afforded by current 
space-borne radar earth observation systems.

\section{A. State of the Art}

Today, several ground-based radar systems are available, operating in $\mathrm{C}[22]-[24], \mathrm{X}$ [25], [26] or $\mathrm{Ku}$ band [22], [23], [27], [28]. The majority of these systems are based on aperture synthesis, using a moving antenna on a rail. An alternative imaging approach is to scan a fan beam by rotating a large antenna [27], [29]. This imaging method has been called type II in [30]. This configuration has certain advantages over groundbased synthetic aperture systems [31] of comparable rail length:

1) Azimuth samples are acquired independently and do not require focusing, eliminating defocusing and loss of coherence caused by moving targets and atmospheric phase screens during aperture time. These changes may adversely affect the coherence of the scene over the aperture length and will worsen the azimuth resolution. This is especially problematic for the analysis of coherent targets.

2) A large angular section of up to $270^{\circ}$ can be imaged in a single pass. This is more difficult to obtain using a rail-based synthetic aperture radar.

An important advantage of rail based SAR systems is the better azimuth resolution: a SAR using a rail of length $L$ will have an angular resolution of:

$$
\theta_{3 d B}^{S A R}=\frac{\lambda}{2 L},
$$

while an antenna with physical aperture size $L$ has an azimuth $3 \mathrm{~dB}$ resolution of:

$$
\theta_{3 d B}^{R A R}=\frac{\lambda}{L}
$$

The majority of polarimetric ground-based radar systems is based on the aperture synthesis principle. An indoor system is presented in [32] followed by a portable outdoors version [33]. A broadband polarimetric SAR system with two dimensional aperture synthesis is introduced in [34], with measurement results presented in [35]. Another example of a synthetic aperture system is described in [25], [26], [36], [36]-[38]. Examples of ground-based polarimetric SAR data at $\mathrm{X}$ and $\mathrm{C}$ band are shown in [24], [39].

A dual polarization, multiband GB-SAR system is used in [40] to produce tomograms of snow covered sea ice. A similar concept is used in [41], [42] to produce tomographic profiles of a snow pack by synthesizing an aperture in the elevation direction.
Excluding non-imaging devices such as ground-based scatterometers, only few real aperture polarimetric ground-based radars exist, one example being the $\mathrm{C}$-band version of the GPRI [43].

\section{B. KAPRI: Real Aperture Polarimetric FMCW Radar}

This paper introduces KAPRI, the Ku-band Advanced Polarimetric Radar Interferometer (KAPRI) [44]. It is an extension of the GPRI (Gamma Portable Radar Interferometer) [27], [29], [45], a real-aperture radar interferometer operating in $\mathrm{Ku}$-band at $17.2 \mathrm{GHz}$. It is designed to monitor unstable slopes using zero baseline differential interferometry [2]; two antennas arranged along a spatial baseline and a dual channel receiver permit to acquire digital elevation models.

The GPRI employs 2 meter long, vertically polarized slotted waveguide antennas, giving the system a $3 \mathrm{~dB}$ azimuth beamwidth of $0.385^{\circ}$ and a $3 \mathrm{~dB}$ elevation beamwidth of $35^{\circ}$.

The feature distinguishing KAPRI and GPRI is the addition of horizontally polarized antennas and switches that permit to connect transmitter and receiver to either type of antenna. Together with modifications in the control software, they enable it to acquire a full polarimetricinterferometric dataset by cycling through all the combinations of transmitted and received polarization during the acquisition.

In Table I the main hardware characteristics of KAPRI are summarized.

\section{Contributions of This Paper}

The following contributions are made in this paper:

1) Preprocessing methods adapted to KAPRIs hardware are presented, that can be used to generate correct SLC images from the acquired raw data.

2) A polarimetric calibration model adapted to the system design of KAPRI is presented. It includes the correction of effects caused by different designs of vertical and horizontal polarized antennas and the presence of spatial baselines between their phase centers.

3) The proposed processing and calibration approaches are validated by analyzing the response of trihedral corner reflectors in a specifically acquired dataset.

\section{Outline}

Section II-A presents the methods employed to process the raw data into range compressed SLC. This part includes a derivation of the FMCW (Frequency Modulated Continuous Wave) signal model and of the 


\begin{tabular}{ll}
\hline Parameter & Value \\
\hline Modulation & FM-CW $(250 \mu s$ to $16 \mathrm{~ms}$ chirp duration) \\
Center frequency & $17.2 \mathrm{GHz}$ \\
Bandwidth & $200 \mathrm{MHz}$ \\
Range resolution & $0.95 \mathrm{~m} \mathrm{3dB}$ resolution @ $-26 \mathrm{~dB}$ peak sidelobe ratio (PSLR) \\
Azimuth 3dB beamwidth & $0.385^{\circ}$ \\
Elevation 3dB beamwidth & $35^{\circ}$ \\
Polarization & fully polarimetric, selectable TX and RX polarization \\
\hline
\end{tabular}

TABLE I: Summary of main KAPRIs parameters.

acquisition geometry, that will be used throughout the rest of this paper. subsection II-B deals with the correction of frequency-dependent beam squint due to the slotted waveguide antenna design. These two sections describe the parts of the processing that are common to both KAPRI and GPRI. The quality of the processing is evaluated in subsection III-A by plotting the oversampled phase and amplitude response of trihedral corner reflectors, where significant range resolution improvements are observed by applying the described squint compensation procedure.

After this step, the range compressed, frequency-squint corrected data still shows a residual azimuth phase variation, especially in the VV channel where a linear variation of almost $30^{\circ}$ is observed for samples inside the antenna beamwidth. This effect is modeled in subsection II-C as a change in distance between the antennas phase center and the scatterers caused by the rotation of the antenna. A method to correct it is proposed and tested in subsection III-B on an array of five trihedral corner reflectors.

An azimuthal shift between the $\mathrm{HH}$ and the VV channel is observed on the response of point targets along with the phase variation; it is ascribed to misaligned antenna patterns. If left uncorrected, it would cause a reduced power and decreased signal-to-noise-ratio (SNR) for crosspolar measurements. To remove it, modified antenna mounts that permit to mechanically shifting the antennas mainlobe were manufactured. They are tested in subsection III-C by analyzing the response of a crosspolarizing dihedral reflector acquired with different antenna mounting settings.

A final step is required before polarimetric calibration: since KAPRI employs separate antennas for each transmit and receive polarization, spatial baselines are obtained between certain combinations of channels. These baselines add a topographic contribution to the polarimetric phase differences. In subsection II-E a method is derived to estimate this contribution using an interferogram obtained from two identically polarized channel on a baseline and rescaled to the undesired polarimetric baseline. Its validity is verified by analyzing the resulting $\mathrm{HH}-\mathrm{VV}$ phase difference in subsection III-D.

In subsection II-F the polarimetric calibration is discussed. A linear distortion model without crosstalk is assumed; the copolar phase and amplitude imbalances are estimated using a trihedral corner reflector, while the imbalance between the crosspolar channel is determined using the $\mathrm{HV}-\mathrm{VH}$ phase difference over distributed scatterers assuming reciprocity. The plausibility of zero cross-talk is assessed by computing the polarization purity of all the trihedral reflectors in the calibration dataset, showing a purity better than $35 d B$ at worst. Finally, in subsection III-E the quality of data calibration is assessed by computing polarization signatures for the trihedral corner reflectors and by estimating calibration model residuals on the corner reflector array.

\section{Methods And DATA}

\section{A. KAPRI: FMCW Radar Signal Model}

A fundamental requirement to generate calibrated polarimetric data is the availability of properly processed SLC images for all elements of the polarimetric scattering matrix. To process them, it is necessary to understand the data acquisition and correct several effects specific to KAPRI. For this purpose a signal model for type II [30] radar data using the deramp-on-receive FMCW architecture [46] is introduced.

Consider a coordinate system having its origin at the location of a radar, as depicted in Figure 1. In this system the antenna is mounted on a lever arm of length $L_{\text {arm }}$; its mainlobe is parallel to the $x$ axis when the pointing angle $\theta$ is 0 . The radar images a scene with a complex reflectivity distribution $\sigma(x, y)$ by measuring range profiles $\hat{\sigma}(R, \theta)$ for a number of antenna azimuths angles $\theta=\arctan \left(\frac{y}{x}\right)$ by rotating the antenna assembly with angular speed $\omega$. Each profile is measured by transmitting a linearly modulated signal of duration $\tau$ with bandwidth $B$ and center frequency $f_{c}$ :

$$
s_{t}(t)=e^{j 2 \pi\left(t f_{c}+\frac{\gamma}{2} t^{2}\right)} .
$$




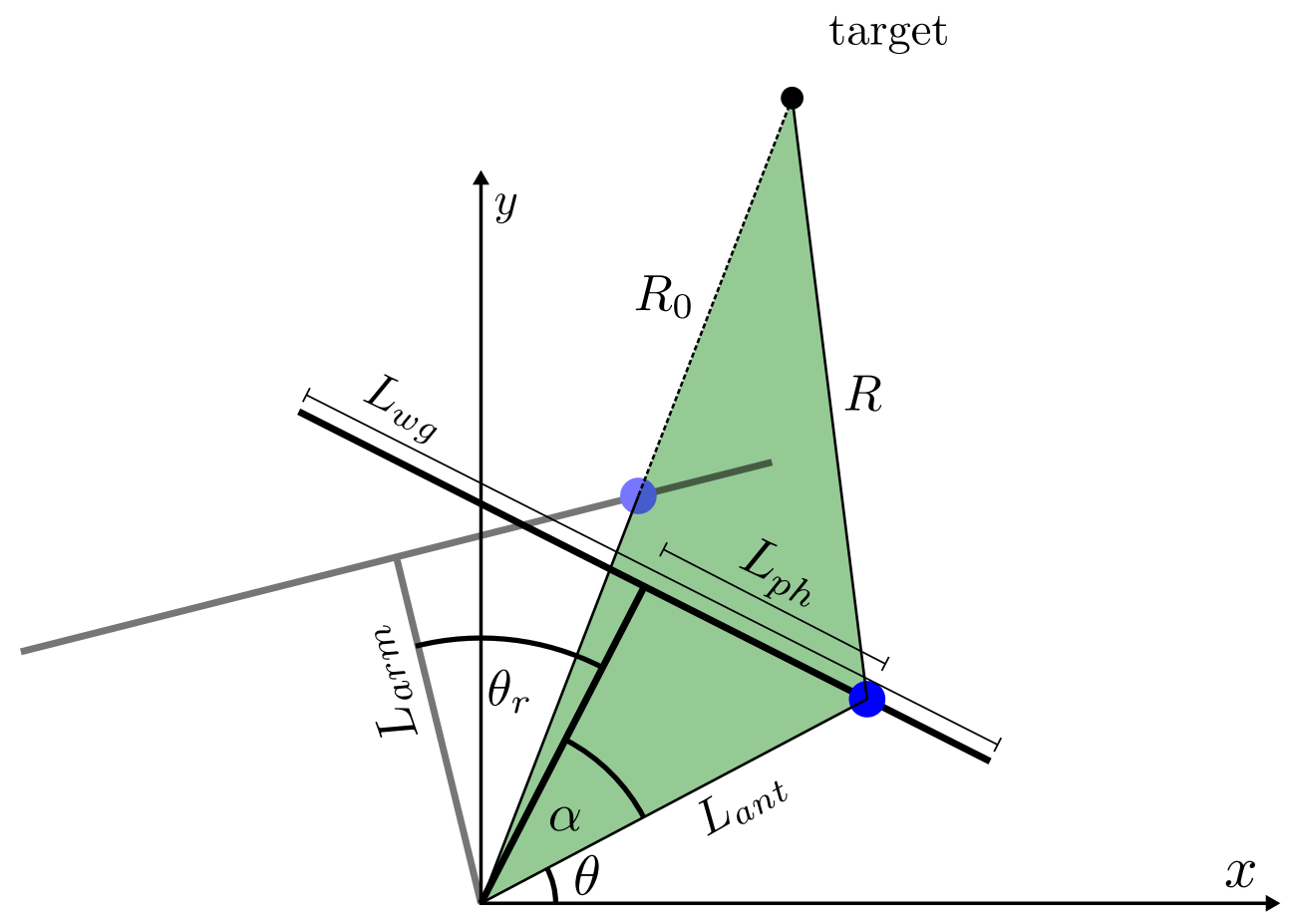

Fig. 1: Geometrical description of the displaced phase center with all relevant parameters, as used in subsection II-C. $R$ is the slant range from the radar to the point scatterer, $L_{p h}$ is the phase center displacement, $L_{w g}$ the length of the antenna, $L_{\text {arm }}$ is the antenna rotation lever arm, $R_{0}$ the range of closest approach and $\alpha$ the additional rotation angle necessary to obtain closest approach when the phase center is not in the midpoint of the antenna but the rotation angle is measured assuming $L_{p h}=0$.

with $\gamma=\frac{B}{\tau}$. The backscattered signal $s_{r}$ for a scatterer at range $R$ is $s_{t}$ delayed by $\frac{2 R}{c}$ and scaled by $\sigma$, the complex reflectivity of the scatterer.

$$
s_{r}(t)=\sigma e^{j 2 \pi\left(\left(t-\frac{2 R}{c}\right) f_{c}+\frac{\gamma}{2}\left(t-\frac{2 R}{c}\right)^{2}\right)} .
$$

In the radar, $s_{r}$ is mixed with the transmitted chirp $s_{t}$ to remove the linear modulation, resulting in a deramped signal $s_{d}$ which is sampled and stored:

$$
\begin{aligned}
& s_{d}(t)=s_{t}(t)^{*} s_{r}(t)= \\
& \sigma e^{j 4 \pi \frac{R}{\lambda}} e^{j 2 \pi f_{b} t} e^{-j 4 \pi \frac{R^{2} \gamma}{c^{2}}} .
\end{aligned}
$$

The deramped signal consist of a phase term with beat frequency $f_{b}=\frac{2 R \gamma}{c}$ which is proportional to the slant range $R$. In addition, the deramped signal contains the following two phase terms:

$e^{j 4 \pi \frac{R}{\lambda}}$-where $\lambda=\frac{c}{f_{c}}$ is the wavelength-is the two way propagation phase, the quantity of interest for interferometric measurements. $e^{-j 4 \pi \frac{R^{2} \gamma}{c^{2}}}$ is proportional to the squared distance, is named "residual video phase" and has to be compensated for in SAR processing.
Although KAPRI is a real-aperture system, an azimuth processing step is required to correct a residual azimuth phase ramp, as described in subsection II-C. However, in this case the variation of $R^{2} B$ during the aperture length is much smaller than $c^{2}$ and the residual video term can be assumed to be zero.

From (5) an by the linearity of the Fourier transform, it follows that the range profile $\hat{\sigma}(R, \theta)$ of a collection of scatterers with complex reflectivity $\sigma_{i}$ at ranges $R_{i}$ can be estimated by the Fourier transform of $s_{d}(t)$.

Using a bandwidth of $200 \mathrm{MHz}$, the theoretical range resolution for KAPRI is $0.75 \mathrm{~m}$ [45]; the effective range resolution is observed to be lower because a Kaiser window is applied to the data before the Fourier transform to mitigate range sidelobes.

To obtain two dimensional images, range profiles are acquired while the antenna is rotated with angular velocity $\omega$. In this case, the deramped signal for a point target at 


$$
\begin{array}{ll}
R, \theta_{t} \text { is: } & \\
& s_{d}(t, \theta)=\sigma e^{j 2 \pi f_{b} t} \\
& e^{j 4 \pi \frac{R}{\lambda}} e^{-j 4 \pi \frac{\gamma R^{2}}{c^{2}}} P\left(\theta-\theta_{t}\right),
\end{array}
$$

where $t$ is the fast time, $\theta=n \omega \tau$ is the azimuth scan angle and $P(\theta)$ describes the two way antenna pattern with beamwidth:

$$
\theta_{3 d B}=\frac{\lambda}{L_{w g}},
$$

where $L_{w g}$ is the size of the antenna aperture and $\lambda$ is the wavelength employed. If the rotation speed is chosen such that $\omega \tau \ll \theta_{3 d B}$, the acquisition is oversampled in azimuth; several range profiles are samples of the same target. Their average in slow time — called azimuth presum or decimation - produces measurements with an increased signal to noise ratio, assuming all range profile to be affected by a white noise process.

Due to diffraction, the radiation beam emitted by the antenna broadens linearly with increasing distance; consequently the spatial resolution in cross-range $\delta_{a z}$ increases with distance:

$$
\delta_{a z}=\frac{R \lambda}{L_{w g}},
$$

\section{B. Beam Squint Correction}

To obtain the desired cross-range resolution at the distances of interest a sufficiently narrow antenna beamwidth is required. To do so, KAPRI employs 2 meter long slotted waveguide antennas [47], [48]. They are constructed by cutting slots resonating at the design frequency in a section of rectangular waveguide. When the slots are appropriately spaced the fields emitted by the cuts combine in phase, producing a narrow beam in the desired direction.

Two types of slotted waveguide antenna exist [49]: the resonant and the traveling wave design. The second type has been chosen because it can be operated with a larger bandwidth; achieving a finer range resolution. The main drawback of this antenna design is its frequencydependent beam squint: when it is operated at frequencies differing from the design value, the phase differences at the slots change; altering the direction of the mainlobe. When a chirped signal is transmitted, the mainlobe direction continuously changes during the pulse. This effect has been used for imaging radars where a mechanical antenna rotation would not be possible [50]-[53]. In the case of KAPRI the beam squint is undesired: the large angular deviation relative to the beamwidth causes the mainlobe to be centered on a scatterer during a fraction of $t_{\text {chirp }}$ only, decreasing the effective transmitted bandwidth and worsening the range resolution.

In dechirped data acquired with sufficient levels of azimuth oversampling, the effect of beam squint is visible as skewed point target responses, as illustrated in Figure 2. During the chirp, the target is only at the center of the beamwidth when the antenna rotation angle matches the beam squint angle. The availability of raw data oversampled in azimuth is key to mitigate the effect of the beam squint:

For each chirp frequency $f$, the data at the corresponding fast time $t_{f}=\frac{f-f_{c}}{\gamma}$ is shifted by the opposite of the squint angle using a bilinear interpolator. The amount of shift is predicted by:

$$
\theta_{s q}=\sin ^{-1}\left(\frac{\lambda}{\lambda_{g_{i j}}}+\frac{k \lambda}{s}\right),
$$

where $\lambda_{g_{i j}}$ is the wavelength for the $i j$-TE mode of the waveguide, $\lambda$ is the free space wavelength, $s$ is the element spacing and $k$ is the mode number. In this case, the waveguide mode used is $T E_{01}$ and $k=0$ is assumed because all the slots must have the same phase [54] to direct the main beam at the antenna broadside.

Instead of using the above expression, a linear approximation for the squint relative to the pointing direction at the design frequency $f_{c}$ is used to process KAPRI data:

$$
\theta_{s q}-\theta_{s q}^{f_{c}}=a f
$$

The equivalent shift to apply at each range line $i_{r}$ in number of azimuth samples $i_{\theta}$ is given by:

$$
i_{\theta}=\frac{a \gamma}{\omega} i_{r}
$$

The linearized expression is necessary because the antenna patterns measurements at different frequencies provided by the manufacturer suggest that the vertically and the horizontally polarized units have different frequency dependent squint behaviors. Because the design information necessary to use the above exact expression for the squint is not available, a data-driven method is be used for the correction of the beam squint.

To estimate the squint rate $a$, the response of a strong point-like target is extracted from the data by windowing it in range and converting it back in the fast time domain with a Fourier transform; its envelope is then extracted with a discrete Hilbert transform. For each frequency the peak is located and stored in a vector from which an estimate of $a$ is obtained by fitting (10).

To correct the frequency-dependent beam squint the azimuth sample spacing must be smaller than the antenna beamwidth: this permits to reconstruct the full bandwidth illumination of each scatterer by combining chirp samples acquired at subsequent azimuth positions. 


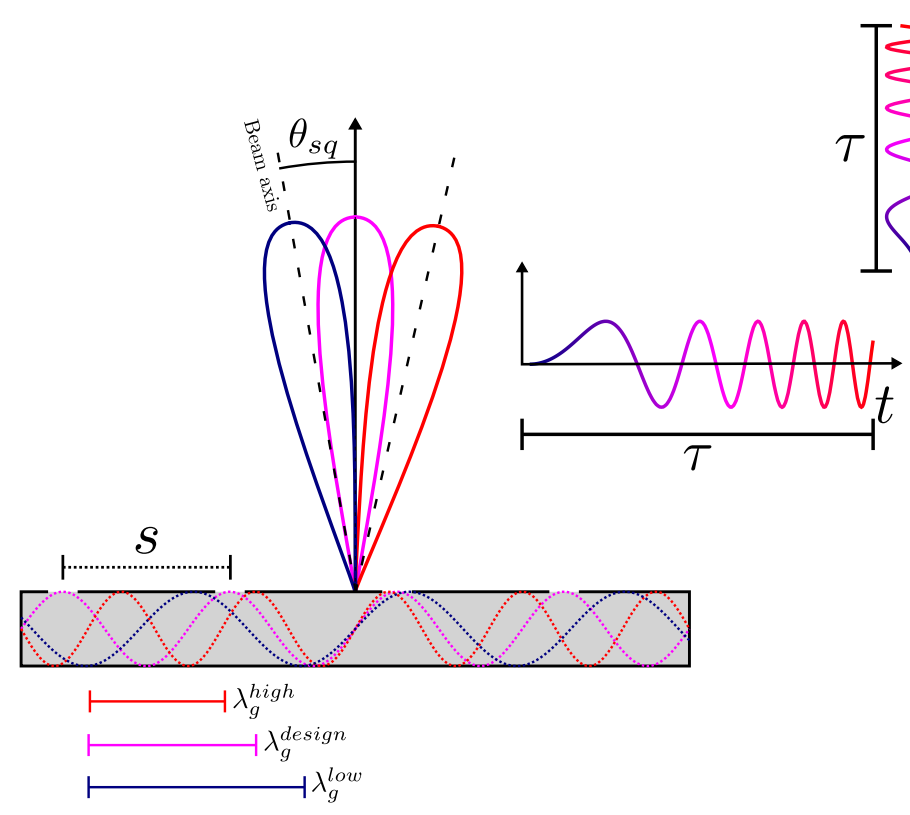

(a)

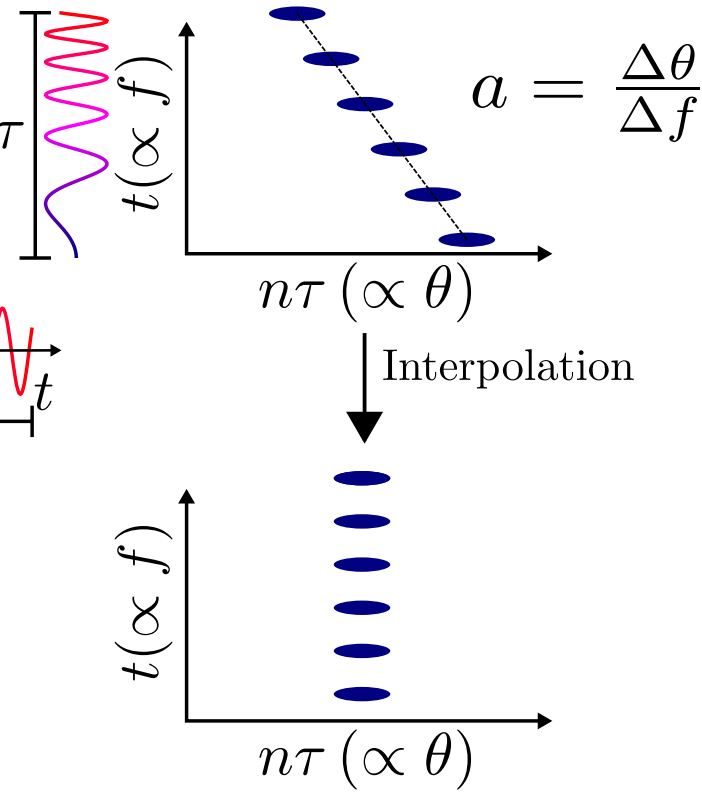

(b)

Fig. 2: (a) Illustration of the frequency-dependent pointing direction $\theta_{s q}$ of the main lobe of the slotted waveguide antennas of KAPRI. The response at different chirp frequencies is represented by the colors of the mainlobes; they correspond to the increasing chirp frequencies as they are represented by the inset plot. The corresponding waveguide wavelengths are displayed underneath the antenna. For the design wavelength $\lambda_{g}^{\text {design }}$, the antenna mainlobe points at the antenna broadside.

(b) The plots represent the time domain envelope of a point target as a function of chirp time $t$ (y-axis) and slow time $n \tau=\frac{\theta}{\omega}$ (x-axis). The $\mathrm{y}$-axis is proportional to the transmitted frequency, the $\mathrm{x}$-axis to the rotation angle of the antenna. If the antennas mainlobe would not change its direction during the chirp, a beat signal as shown in the bottom would be obtained. It would have a frequency proportional to the range and would cover the slow time extent where the objects stays within the beamwidth. In the case when the mainlobe changes direction with frequency, the response is skewed because the target is only illuminated at the moment when the mechanical and the electrical antenna pointing direction match, causing its response to occupy several azimuth cells. After range compression, obtained with a Fourier transform along the $t$ axis, the frequency-dependent antenna pointing would cause a loss of range resolution because only a fraction of the transmitted bandwidth would be used for each azimuth bin. To correct this effect, the data is interpolated in the time domain in order to reconstruct the full bandwidth by combining the subsequent sub-bandwidths that are obtained at different mechanical pointing angle. This correction requires sufficient azimuth oversampling, which is achieved by slow rotation of the antennas w.r.t the chirp duration.

After correcting the beam squint, azimuth presumming can be applied if desired to reduce the data size and increase SNR.

Then, an Hann window is applied to the first and last $z$ samples of the raw data $s_{d}$ to mitigate the transient signal caused by the abrupt change in frequency due to the repetition of the chirp and the end of each pulse. A Kaiser window is applied to reduce range processing sidelobes that are caused by the finite bandwidth. Finally, a fast time Fourier transform performs the range compression to obtain the SLC image $\hat{\sigma}(R, \theta)$.

Each range line of the SLC is then multiplied by $\sqrt{R^{3}}$ to compensate for the power spreading loss. In this manner, the intensity of the SLC data is directly proportional to the radar brightness $\beta_{0}$ [55].

\section{Azimuth Processing}

In the case of KAPRI, the correction of frequencydependent squint as described in the preceding section is not sufficient to produce correct SLC data: a strong residual azimuth phase is observed on the response of point-like scatterers, especially in the VV channel. This phase contribution is problematic for two reasons: 
1) Because the azimuth samples have differing phases it reduce the amplitude and the SNR after azimuth presumming.

2) Since each polarization displays a different ramp, the coherence magnitude of polarimetric phase differences will be reduced and the resulting coherence phase will be affected by a residual phase variation. For example, if the HH-VV phase is needed for calibration in the method described in subsection II-F and the azimuth variation is not taken into account, the additional phase will results in incorrect calibration parameters.

The azimuthal phase variation arises from the rotational acquisition: the antennas are mounted with an offset $L_{\text {arm }}$ from their center of rotation, as shown in Figure 1. Therefore, during the rotation the distance from the phase center to a scatterer varies with the azimuth position, changing the propagation phase accordingly [56]. If the variation in distance is larger than the range resolution, the target will move though several range cells as a function of azimuth position, in analogy to range cell migration in SAR systems. In the case of KAPRI the variation in distance is two orders of magnitude smaller than the range resolution and will only visibly affect the phase of the signal.

To model the change in phase, consider the radar acquisition geometry depicted in Figure 1:

The antennas are mounted on a lever arm offset by $L_{\text {arm }}$ from the center of rotation. Their phase centers have an horizontal displacement $L_{p h}$ from the lever arm attachment. A point target is considered, located at the range of closest approach $R_{0}$, obtained when the phase center, the target and the lever arm are aligned. For a incremental rotation $\theta_{r}$ from the angle of closest approach, the relative phase variation, is:

$$
\phi_{\text {scan }}=\frac{4 \pi}{\lambda}\left(R_{0}-R\left(\theta_{r}\right)\right) .
$$

Where $R$ is the effective distance between the point target and the phase center, computed with the law of cosines on the green triangle of Figure 1 with the included angle $\theta_{r}$, one side $L_{a n t}=\sqrt{L_{a r m}^{2}+L_{p h}^{2}}$ and the other $c=L_{\text {ant }}+R_{0}$ :

$$
R\left(\theta_{r}\right)=\sqrt{c^{2}+L_{\text {ant }}^{2}-2 c L_{a n t} \cos \left(\theta_{r}-\alpha\right)} .
$$

$L_{a n t}$ is interpreted as the equivalent lever arm of an antenna without the horizontal phase center shift that would produce the same phase variation as a system with a nonzero $L_{p h}$.

The function is shifted by the angle $\alpha=\arctan \left(\frac{L_{p h}}{L_{a r m}}\right)$ that describes the additional rotation needed for an antenna with nonzero $L_{p h}$ to be at closest approach. This shift is necessary because the azimuth angle is measured assuming a zero $L_{p h}$ : the reading given by the angle encoder does not correspond to the angular position of the phase center.

The complete characterization of the phase ramp requires the knowledge of the antenna phase center displacement $L_{p h}$. Normally, it is assumed that the phase center is at the midpoint of the antenna. However, when the experimental data was analyzed using this assumption (which implies $L_{a n t}=L_{a r m}$ ), (13) failed to model the observed azimuth phase variation and possibility of a displaced antenna phase center was included. This displacement is used to describe physical differences in the antennas, which could explain the larger azimuth phase in the VV channel.

$L_{p h}$ is estimated using the measured phase of a point target by minimizing the squared distance with the phase simulated according to (13).

$$
\hat{L}_{p h}=\underset{\left(L_{p h}, \phi_{o f f}\right)}{\operatorname{argmax}}\left\|\phi_{\text {meas }}-\phi_{\text {scan }}\right\|^{2},
$$

where $\phi_{\text {sim }}=\phi_{\text {scan }}+\phi_{\text {off }}$ is the simulated phase with an additional offset that accounts for noise and the intrinsic scattering phase and the system.

When $L_{p h}$ is known, the azimuth phase variation is corrected in the range compressed data $\hat{\sigma}$ by convolution of each range $R_{i}$ with the phase of (13) used as a matched filter:

$$
\begin{aligned}
\hat{\sigma}^{\text {corr }}\left(R_{i}, \theta\right)= & \int_{-\frac{\theta_{i n t}}{2}}^{\frac{\theta_{\text {int }}}{2}} e^{-\jmath \frac{4 \pi}{\lambda} R\left(\theta-\theta^{\prime}, R_{i}\right)} \\
& \hat{\sigma}\left(R_{i}, \theta^{\prime}\right) d \theta^{\prime} .
\end{aligned}
$$

This expression replaces the azimuth presum described in subsection II-A: the samples are now averaged with an appropriate phase factor; this combines SNR improvement and the correction of the azimuth trend in a single step. Because the model only describes the variation of the phase relative to closest approach $R_{0}$, the absolute phase is preserved; this is very important for interferometry and polarimetry.

The procedure is similar to azimuth focusing of synthetic aperture data, where the cross-range resolution is obtained by the integration of the data in the azimuthtime direction. However, in the case of real aperture systems the resolution is limited by the physical antenna beamwidth and the response of a target occupies a single azimuth sample; unless the data is oversampled. In the first case, integrating the data in azimuth degrades the resolution because samples that do represent the same 
scatterer are combined.

To correct the phase variation without an excessive increase in azimuth resolution, the integration is limited to a window of size $\theta_{\text {int }}$. The optimal trade-off is empirically determined to be $0.6^{\circ}$, slightly larger than $\theta_{3 d B}$.

\section{Antenna Pattern Misalignment}

By analyzing the response of a strong point-like target a significant azimuth shift between the $\mathrm{HH}$ and the $\mathrm{VV}$ channels was observed, corresponding to an azimuth pattern mispointing of $0.2^{\circ}$, almost half of the antenna beamwidth. The misalignment is particularly problematic for cross-polar measurements: the transmitting and receiving patterns are not aligned. Using the available pattern information a power loss of approx $2.5 \mathrm{~dB}$ compared to the ideal case is predicted. This loss reduces the SNR for the cross-polar channels, leading to noisier measurements.

While the offset between copolar channels can be corrected by coregistration, no a posteriori method can compensate the SNR loss in the crosspolar measurements.

To realign the patterns, an adjustable antenna mount was manufactured by replacing one of two hinges where the antennas are fixed on the tower (see Figure 3) with an adjustable bracket that allows to slide the antenna back and forth on the one side, obtaining the effect of rotating it around the center. Based on the size of the antenna mounting bracket and on the amount of misalignment, it was determined that the horizontally polarized antennas need to be shifted by $1.8 \mathrm{~mm}$ to align the antenna patterns.

\section{E. Removal of Topographic Phase}

The next step on the way to calibrated polarimetric data requires a brief review of KAPRIs antenna configuration, depicted in Figure 4:

Six antennas are mounted on a supporting structure connected to the rotary scanner. Of these, 2 are transmitting antennas, one for each polarization. The remaining 4 are connected in pairs through switches to the receivers; each pair composed of an horizontally and a vertically polarized unit. This configuration permits to acquire full polarimetric dataset by selecting the desired antennas for the transmitter and for each receiver separately. This arrangement ensures a low level of polarimetric crosstalk because only one combination is acquired at each time and the antennas are physically separated. Additionally, the separation of transmitting and receiving antennas increases the transmit-receive isolation, a fundamental requirement for FMCW performance [45], [46], [57].

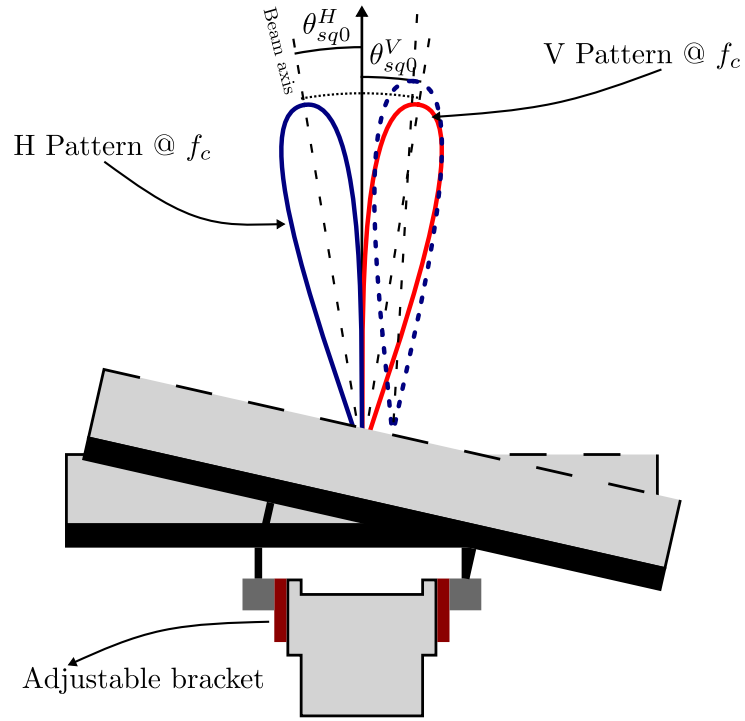

Fig. 3: Illustration of the adjustable antenna mount allowing to shift the patterns to bring the $\mathrm{H}$ and the $\mathrm{V}$ antennas into alignment. The left bracket can be slid towards the front, allowing the antenna to pivot on the right hinge. For small shifts, this movement approximates a rotation around the center of the antenna tower, as depicted by the blue antenna pattern.

The disadvantage of this configuration is that certain combinations of channels $i$ and $j$ will be separated by a baseline: These antennas can be replaced by an equivalent antenna located at the midpoint between transmitter and receiver [37]. For the combinations of polarizations $i$ and $j$ where the equivalent phase centers are separated by a baseline $\mathbf{b}_{i j}^{e q}$, the polarimetric phase difference $\phi_{i j}$ will contain an interferometric contribution:

$$
\phi_{i j}=\phi_{i j}^{p o l}+\phi_{i j}^{p r o p}
$$

This term appears as topographic fringes in the polarimetric phase difference. It will complicate calibration by adding an additional phase contribution unrelated to the polarimetric properties of the scatterer.

To obtain correct phase differences the topographic contribution can be estimated by considering two additional channel $k$ and $l$ acquired with a non-zero baseline $\mathbf{b}_{k l}^{e q}$ and with the same polarization, where $\phi_{k l}^{p o l}=0$. In this case, the propagation phase difference can be approximated as a function of the local incidence angle and of the perpendicular baseline separating the phase centers:

$$
\phi_{k l}^{p r o p}=\frac{4 \pi}{\lambda} b_{k l}^{e q} \sin \left(\theta_{k l}^{l}-\alpha_{k l}^{b l}\right),
$$




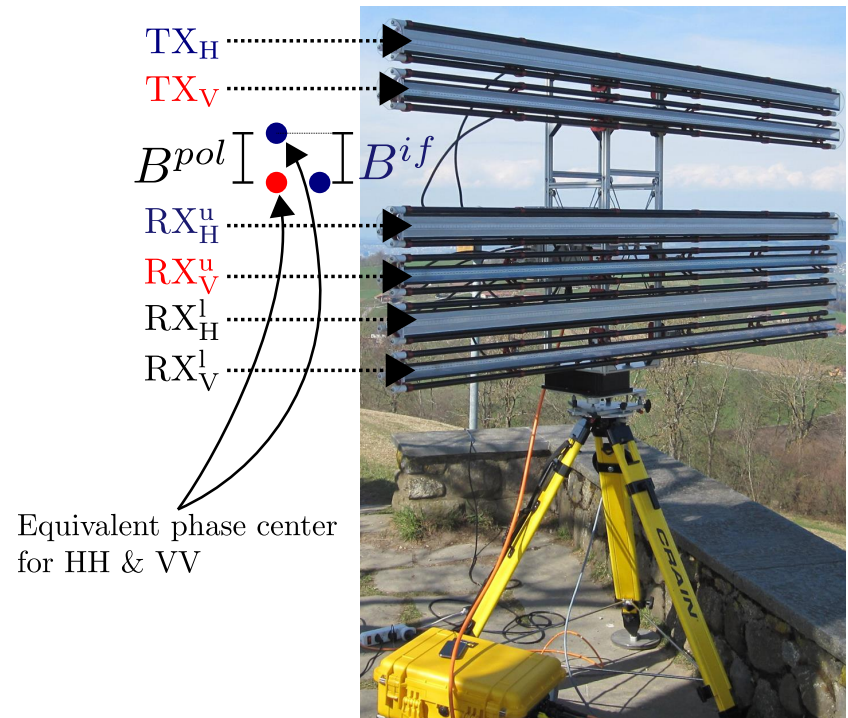

Fig. 4: KAPRI radar during a field test. The antenna arrangement used in this paper is overlaid. The blue and red dots represent the equivalent phase centers for the $\mathrm{HH}$ and $\mathrm{VV}$ channels from the top receiver. The bottom blue dot on the right represents the one for the lower $\mathrm{HH}$ channel, which is used in conjunction with the upper $\mathrm{HH}$ phase center to estimate the topographic phase contribution.

where $\alpha_{k l}^{b l}$ is the baseline angle w.r.t to the vertical, the look angle $\theta_{l}$ is the angle between the line of sight vector and the vertical, $b_{k l}^{e q}$ is the perpendicular baseline between the equivalent phase centers. Then $\phi_{i j}^{\text {prop }}$ can be estimated from $\phi_{k l}$ if the look vector elevation angle and the baseline orientation do not significantly change from $k l$ to $i j$, i.e if $\theta_{i j}^{l}-\alpha_{i j}^{b l} \approx \theta_{k l}^{l}-\alpha_{k l}^{b l}$.

$$
\hat{\phi}_{i j}^{\text {prop }}=\frac{b_{i j}^{e q}}{b_{k l}^{e q}} \phi_{k l} .
$$

if the ratio of the baselines is not integer [58] phase unwrapping of $\phi_{k l}^{\text {prop }}$ is required before rescaling.

To correct all combinations that have a non-zero baseline, the measured scattering matrix $\mathbf{S}$ is converted into a covariance matrix $\mathbf{C}$; then $\hat{\phi}_{i j}^{\text {prop }}$ is subtracted from the phase of every non-diagonal element $i j$ where $b_{i j}^{e q} \neq 0$. The result is a terrain flattened covariance matrix where the phase of off-diagonal elements only contains polarimetric contributions, possibly affected by an offset that needs compensation in the polarimetric calibration.

\section{F. Polarimetric Calibration}

The polarimetric calibration is based on the covariance matrix corrected above; the procedure is based on the linear distortion matrix model [59], [60] relating the observed scattering matrix $\mathbf{S}^{\prime}$ with the theoretical $\mathbf{S}$ :

$$
\mathbf{S}^{\prime}=\mathbf{R S T} \text {. }
$$

The same can be restated for polarimetric covariance matrices $\mathbf{C}=\mathbf{S S}^{H}$ :

$$
\mathbf{C}^{\prime}=\mathbf{D C D}^{H} .
$$

where $\mathbf{D}$ is the Kronecker product of $\mathbf{R}$ and $\mathbf{T}$, the matrices that describe the phase and amplitude imbalances and crosstalk in reception and transmission.

In the case of KAPRI, crosstalk calibration is not considered: the radar has a good polarization isolation, largely because only one polarization is acquired at a time by selecting the appropriate combination of transmitting and receiving antennas. The only source of crosstalk is the presence of cross-polarized antenna sidelobes in the direction of the antenna mainlobe. The manufacturer has provided simulated radiation patterns for the horizontally polarized antennas, where the isolation between the co and the cross polarized pattern in the main-lobe direction is observed to be better than $60 \mathrm{~dB}$. Additionally, by computing the VV-HV ratio of the oversampled response of a trihedral corner reflector, the polarization purity of the system was estimated to be better than $35 \mathrm{~dB}$ in the worst case.

Under the assumption of negligible crosstalk, the distortion matrices are:

$$
\begin{aligned}
& \mathbf{R}=A\left[\begin{array}{cc}
1 & 0 \\
0 & f / g e^{i \phi_{r}}
\end{array}\right], \\
& \mathbf{T}=A\left[\begin{array}{cc}
1 & 0 \\
0 & f g e^{i \phi_{t}}
\end{array}\right],
\end{aligned}
$$

where $f$ is the one-way copolar amplitude imbalance relative to the $\mathrm{H}$ polarization, and $g$ the amplitude imbalance of the crosspolarized channels. $\phi_{t}=\phi_{t, h}-\phi_{t, v}$ is the phase offset between the polarizations when transmitting, $\phi_{r}=\phi_{r, h}-\phi_{r, v}$ is the phase offset in reception and $A$ is the absolute amplitude calibration parameter [61], [62].

The four complex parameters in $\mathbf{D}$ can be estimated using a trihedral corner reflector and a reciprocal scatterer with a significant cross polarized contribution [37], [63]. The copolar amplitude imbalance $f$ is estimated by the ratio of $C_{H H H H}^{\prime}$ and $C_{V V V V}^{\prime}$ :

$$
f=\left(\frac{C_{V V V V}^{\prime}}{C_{H H H H}^{\prime}}\right)^{\frac{1}{4}} .
$$


The copolar phase imbalance $\phi_{r}+\phi_{t}$ is estimated from the phase of $C_{V V H H}^{\prime}$ :

$$
\phi_{r}+\phi_{t}=\arg \left(C_{V V H H}^{\prime}\right) .
$$

Both parameters are estimated on the oversampled response of a corner reflector. In [62], a polynomial model for the copolar calibration parameters is used, that links the imbalances to the incidence angle. In that case, the estimate are performed using an array of corner reflectors covering a large range of incidence angles. In the case of this paper, such an array is not available and a simplified calibration model is used, assuming the copolar parameters to be independent of incidence angle. Because of the difficulty of placing and correctly orienting a cross-polarizing target such as a $45^{\circ}$ dihedral, the estimation of $g$ and $\phi_{t}-\phi_{r}$ is based on the assumption that most pixels in the data represent reciprocal scatterers:

$$
g=\left\langle\frac{C_{H V H V}^{\prime}}{C_{V H V H}^{\prime}}\right\rangle^{\frac{1}{4}}
$$

and:

$$
\phi_{t}-\phi_{r}=\arg \left(\left\langle C_{V V H V}^{\prime}\right\rangle\right) .
$$

Finally, calibrated covariance matrices are obtained by inverting (20) using the estimated D.

If radiometric calibration is desired, the value of $A$ can be determined after imbalance correction from the ratio of the expected and the measured RCS (Radar Cross Section).

$$
A=\left(\frac{\sigma_{t r i}}{C_{H H H H}^{\prime} R_{t r i}^{3}}\right)^{\frac{1}{2}} .
$$

Where $R_{t r i}$ is the slant range to the trihedral corner reflector. This is necessary to remove the range spread loss compensation as performed in section II.

\section{G. Experimental Data}

A dataset for calibration purposes was acquired in September 2016 at an urban-agricultural area near Münsingen, Switzerland. The data was acquired from the top of the "Chutzen" hill approximately $800 \mathrm{~m}$ a.s.1, looking down towards a mixture of fields and forests, with the town in the far range region. Six trihedral corner reflectors were placed in the area for the determination of calibration parameters and to assess imaging quality. Three of these reflectors have triangular faces with a length of $40 \mathrm{~cm}$, corresponding to a RCS of $\frac{4}{3} \pi \frac{a^{4}}{\lambda^{2}}=25.5 \mathrm{~dB}$, while the remaining two are cubic corner reflector with a RCS of $35 \mathrm{~dB}$, at the nominal central frequency of $17.2 \mathrm{GHz}$. Figure 5 shows the calibrated Pauli RGB composite of the scene, interpolated

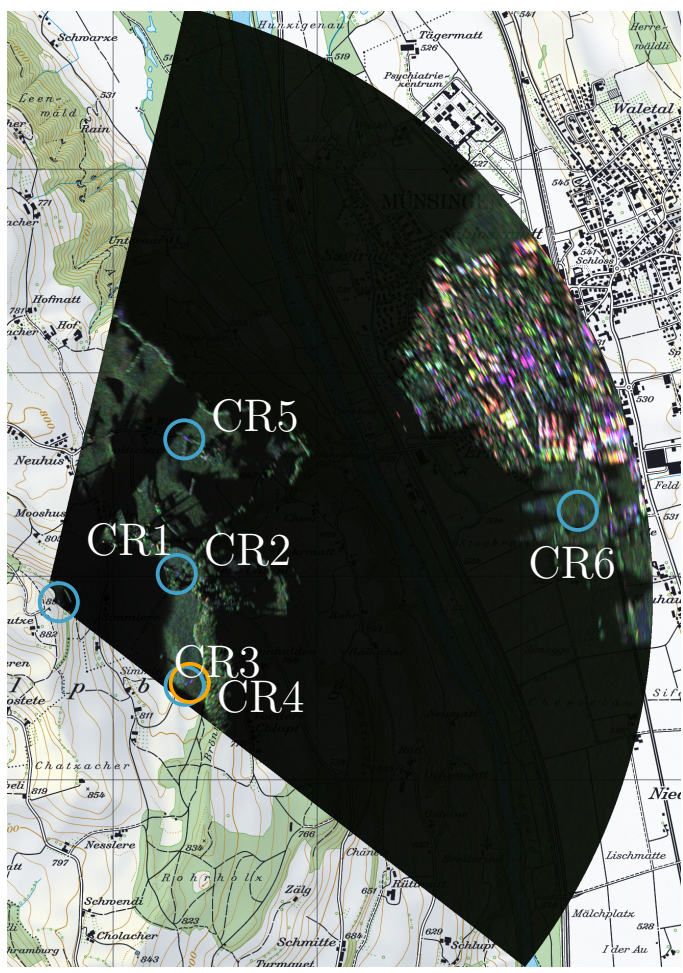

Fig. 5: Pauli RGB composite $(R=|H H-V V|, G=$ $|H V|, B=|H H+V V|)$ of the imaged scene, geocoded using a digital elevation model with $2 \mathrm{~m}$ pixel spacing. Each channel is scaled according to its own dynamic range. The location of corner reflectors is marked by cyan circles, the reflector used for polarimetric calibration is shown in orange. The image is overlaid on a 1:25000 scale Swiss topographic map (Reproduced with the authorization of swisstopo JD100042).

in Cartesian coordinates using a $2 \mathrm{~m}$ posting DEM. The location of the reflectors is marked using blue circles and their names according to Table II are shown next to them.

The dataset was acquired with the horizontally polarized antenna group shifted towards the $\mathrm{V}$ group by $1.8 \mathrm{~mm}$ to compensate for the pattern misalignment as described in subsection II-D.

A second dataset containing a dihedral reflector was acquired at ETH Hönggerberg campus in order to investigate the effect of antenna pattern misalignment on crosspolar acquisitions and to test the suitability of the computed adjustment value as discussed in subsection II-D. This measurement was held separately because of the logistical problems associated with the transportation and the setup of large dihedral calibration 
targets.

\begin{tabular}{cccc}
\hline Name & $R_{0}[\mathrm{~m}]$ & $\sigma_{0}[\mathrm{~dB}]$ & Type \\
\hline CR1 & 74.3 & 35 & cubic \\
CR2 & 673.1 & 25.5 & triangular \\
CR3 & 824.6 & 35 & cubic \\
CR4 & 838.9 & 25.5 & triangular \\
CR5 & 1,049 & 35 & cubic \\
CR6 & $2,689.3$ & 25.5 & triangular \\
\hline
\end{tabular}

TABLE II: Summary of TCRs in the calibration dataset: Name of nearest map feature, Distance from the radar, expected radar cross section, type of reflector.

\section{RESULTS}

\section{A. Beam Squint Correction}

Figure 6 shows the frequency-azimuth response relative to the pointing at the center frequency for the "CR2" TCR. This plot was generated with the procedure described in subsection II-B by filtering the range compressed data around the location of the reflector and converting it back to the time domain by an inverse Fourier transform. In (a) and (c), the procedure was applied to the $\mathrm{HH}$ and $\mathrm{VV}$ channel data before any squint correction was applied. In (b) and (d) the same is shown after applying the interpolation described in subsection II-B using $a=4.2 \frac{\circ}{G H z}$ and $a=3.9 \frac{\circ}{G H z}$ for the $\mathrm{HH}$ and VV channels. In each plot, a line shows the results of the linear model fit described by (10), the estimated $a$ parameter is overlaid to the plot.

The same fit procedure is applied to all reflectors for both the $\mathrm{HH}$ and the VV data; the results are shown in Table III.

In Figure 7, panels (a),(b), (d) and (e) the effect of frequency dependent squint and the result of its correction are visible for the "CR2" reflector. The plots are generated by oversampling the range compressed data around the reflector in azimuth and range using a cubic order spline approximating a sinc interpolator. In each plot azimuth and range resolutions are estimated numerically by fitting a spline on the response at the corresponding maximum location and computing its 3 $\mathrm{dB}$ width. Panels (a) and (d) show the range compressed data for the $\mathrm{HH}$ and VV channels. In (b) and (e) the same is repeated after applying squint correction before range compression.

\section{B. Azimuth Processing}

The ability of the phase model described in subsection II-C to explain the observed phase variation on the $V V$ channel response is tested on each reflector in the array: the maximum in range and azimuth was identified and the samples corresponding to the half power beamwidth were extracted at the range of maximum intensity. The unwrapped phase is used to estimate $L_{p h}$ according to (14).

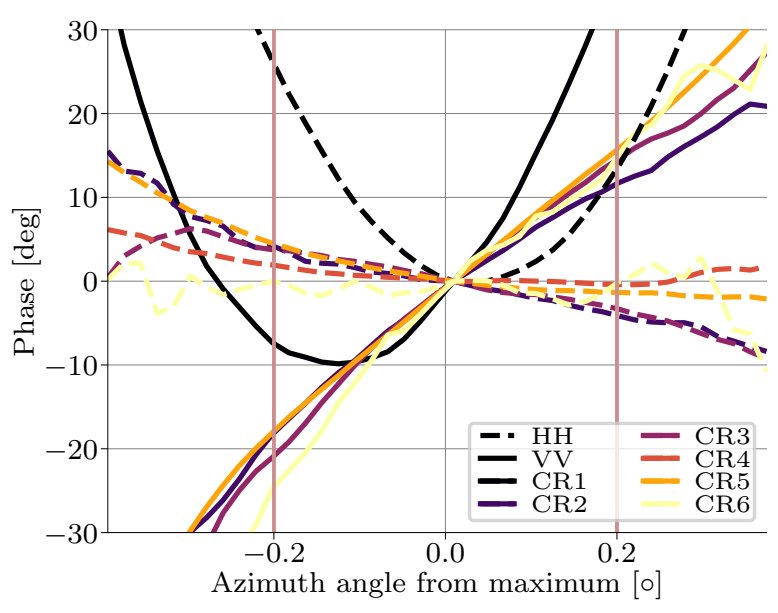

(a)

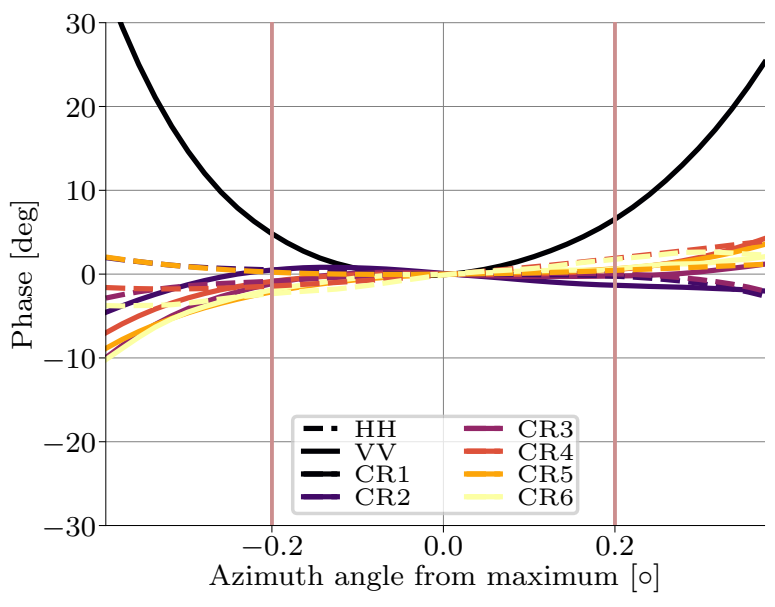

(b)

Fig. 8: Relative phase response for all reflectors in the calibration array, (a) no azimuth phase correction (b) after azimuth phase correction. Continuous lines: VV channel, dashed lines: HH channel. To display the relative phase variation, the phase at the maximum is subtracted from each plot. The vertical lines indicate the theoretical $3 \mathrm{~dB}$ resolution of the antenna $\theta_{3 d B}$.

The resulting model parameters fit values for the $\mathrm{H}$ and $\mathrm{V}$ antennas are shown in Table IV alongside with the distance from the radar and the name of the reflector, defined in Table II. Owing to the lack of sufficiently bright 


\section{CR2}

(a)

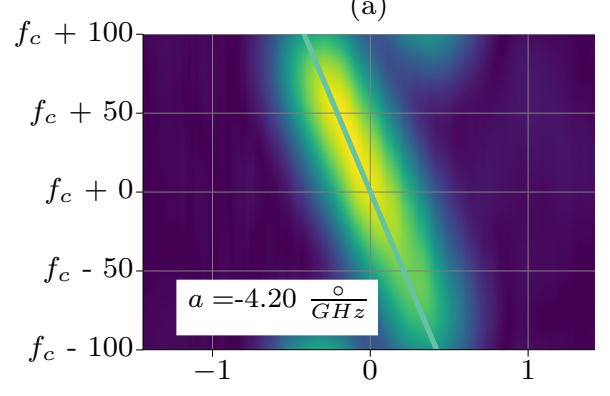

(b)

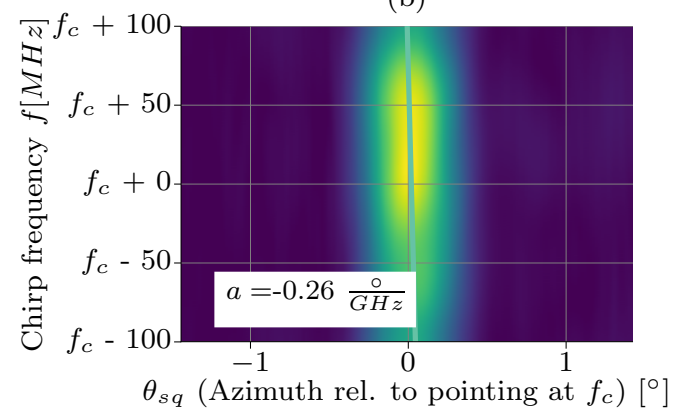

(c)

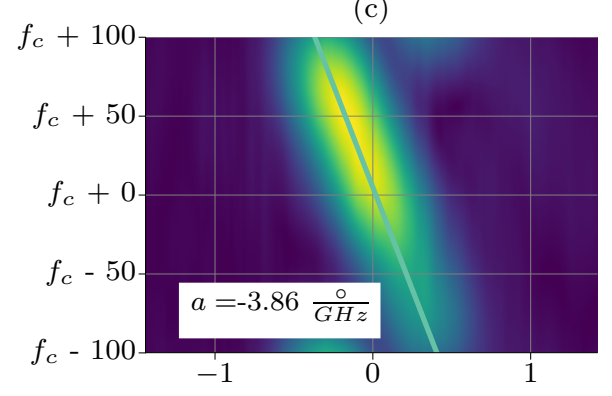

(d)

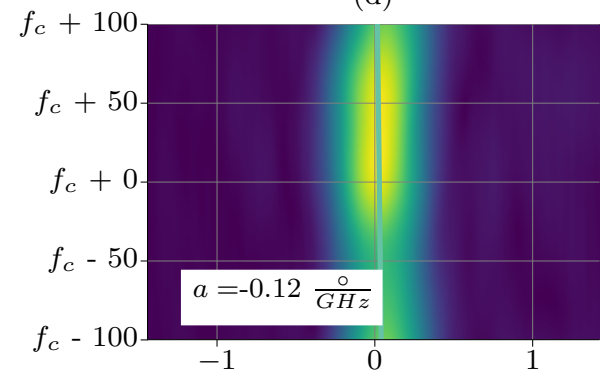

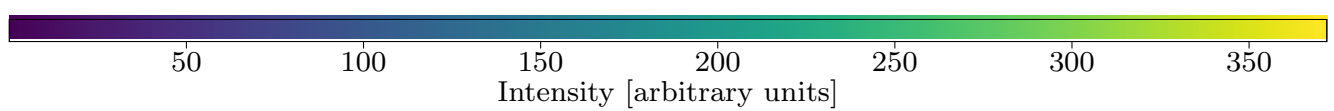

Fig. 6: Azimuth-frequency response of the "CR2" TCR: the raw data samples around the reflectors azimuth location were extracted, then filtered in range by Fourier transforming them along the frequency axis, applying an Hamming window about the range location and converting them back into the time domain with an inverse Fourier transform. By doing so, only the portion of the range spectrum close to the reflectors location was kept. Finally, the complex envelope of the data was extracted using a discrete Hilbert transform. This is conceptually equivalent to the plot of Figure 2. (a) for the HH channel, (b) for the HH channel after the interpolation described in subsection II-B, (c) for the VV channel, and (d) the same as (b) for the VV channel.

\begin{tabular}{ccccc}
\hline Name & $a_{H H}$ & $a_{V V}$ & residual $a_{H H}$ & residual $a_{V V}$ \\
\hline CR1 & $-3.7 \cdot 10^{-9}$ & $-4.1 \cdot 10^{-9}$ & $-1.1 \cdot 10^{-10}$ & $-8.3 \cdot 10^{-10}$ \\
CR2 & $-4.2 \cdot 10^{-9}$ & $-3.9 \cdot 10^{-9}$ & $-2.6 \cdot 10^{-10}$ & $-1.2 \cdot 10^{-10}$ \\
CR3 & $-3.9 \cdot 10^{-9}$ & $-3.7 \cdot 10^{-9}$ & $-4 \cdot 10^{-11}$ & $1.4 \cdot 10^{-11}$ \\
CR4 & $-4 \cdot 10^{-9}$ & $-3.7 \cdot 10^{-9}$ & $-7.9 \cdot 10^{-11}$ & $-2.8 \cdot 10^{-10}$ \\
CR5 & $-4.2 \cdot 10^{-9}$ & $-4 \cdot 10^{-9}$ & $-2 \cdot 10^{-10}$ & $-2.4 \cdot 10^{-10}$ \\
CR6 & $-3.9 \cdot 10^{-9}$ & $-3.9 \cdot 10^{-9}$ & $-1.4 \cdot 10^{-10}$ & $-4.2 \cdot 10^{-10}$ \\
mean & $-4 \cdot 10^{-9}$ & $-3.9 \cdot 10^{-9}$ & $-1.4 \cdot 10^{-10}$ & $-3.1 \cdot 10^{-10}$ \\
\hline
\end{tabular}

TABLE III: Result of fitting the model of (10) to each reflector in the calibration array. In the second column, the $a$ parameter for the HH channel is shown, in the third the one for the VV channel. The last two columns show the same parameters re-estimated after applying the squint correction using 4.2 and $3.9 \frac{\circ}{G H z}$ for $a_{H H}$ and $a_{V V}$ respectively.

crosspolarizing point targets, the equivalent horizontal phase center locations for $\mathrm{HV}$ and $\mathrm{VH}$ channels were not directly estimated from the data. In the following, their location it assumed to be at the midpoint between $L_{p h}^{H}$ and $L_{p h}^{V}$, the theoretical equivalent phase center for these channels. 
(a)

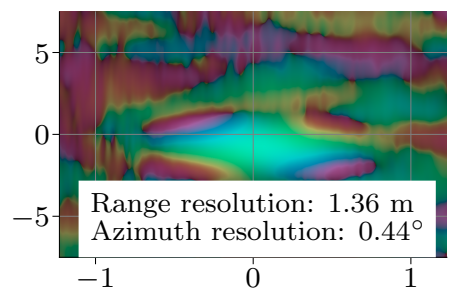

(d)

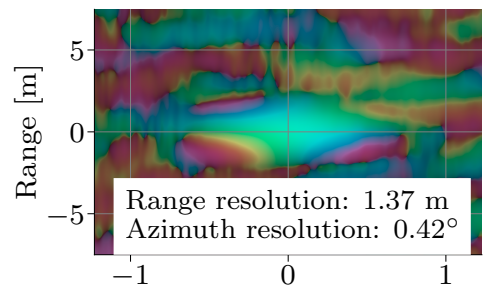

(b)

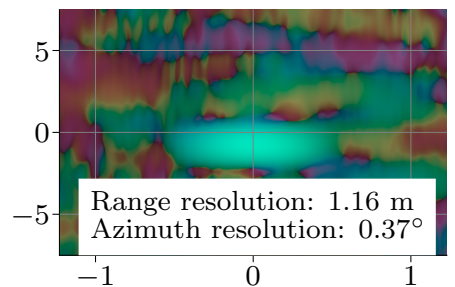

(e)

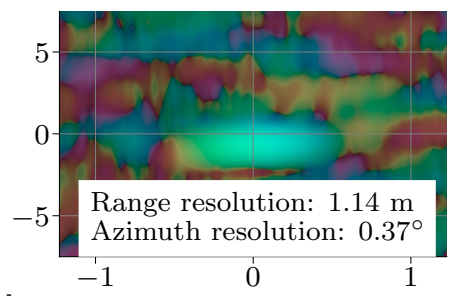

(c)

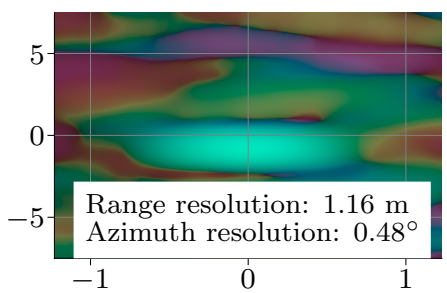

Azimuth angle from maximum [o]

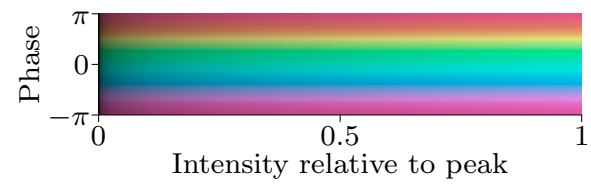

Fig. 7: Oversampled phase and amplitude responses for the corner reflector "CR2" at $673 \mathrm{~m}$ slant range. (a) HH channel without correction, (b) HH channel with frequency-dependent squint compensation (c) same as (b) with azimuth phase ramp removal. (d) VV channel without correction, (e) VV channel with frequency-dependent squint compensation, (f) same as (e) with azimuth phase ramp removal. (g) Color palette used to represent the above plots. The hue is modulated by the phase, while the brightness corresponds to the gamma-corrected normalized intensity. The phase of each response is referenced to the phase at the peak.

The result of applying the correction of (15) to the TCR "CR2" at $673 \mathrm{~m}$ is displayed in panels (c) and (f) of Figure 7.

In Figure $8 \mathrm{~b}$ the phase response in both the $\mathrm{HH}$ and

\begin{tabular}{cccc}
\hline Name & $R_{0}[m]$ & $L_{p h}^{H}[m]$ & $L_{p h}^{V}[m]$ \\
\hline CR1 & 74.3 & 0.03 & -0.18 \\
CR2 & 673.1 & 0.02 & -0.1 \\
CR3 & 824.6 & 0.03 & -0.12 \\
CR4 & 838.9 & 0.01 & -0.12 \\
CR5 & 1,049 & 0.02 & -0.12 \\
CR6 & $2,689.3$ & 0 & -0.12 \\
\hline
\end{tabular}

TABLE IV: Result of the phase center displacement fit for six trihedral corner reflectors located at different ranges. In the thirds column, the estimated phase center displacements for the $\mathrm{H}$ antenna are shown, in the fourth the ones for the $\mathrm{V}$ unit.

VV channels is plotted for all reflectors. To produce the plot, all responses were aligned in azimuth, normalized to the maximum, finally their phase was referenced to the phase at closest approach; this corresponds to the phase of (12). This allows an easier comparison of the azimuthal phase variation.

\section{Antenna Pattern Misalignment}

To verify the impact of the H-V pattern pointing shift as described in subsection II-D on the performance of crosspolar measurements, the response of a dihedral reflector with an high crosspolar contribution is analyzed for two configurations:

1) the antennas are not mechanically moved; the $H$ and $\mathrm{V}$ pattern are not aligned in azimuth.

2) the optimal shift of $1.8 \mathrm{~mm}$, as described in subsection II-D is applied to the movable antenna hinge to bring the patterns into alignment. 


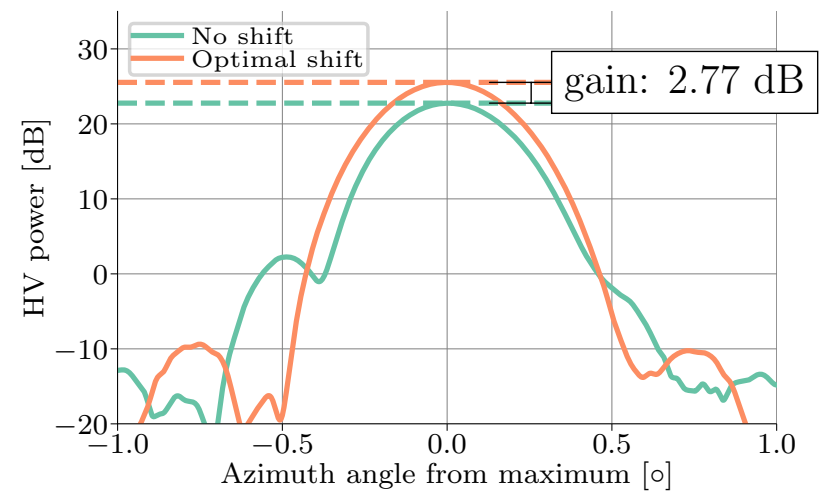

Fig. 9: Oversampled azimuth power response of a dihedral corner reflector, before (green line) and after the correction of antenna pattern mispointing (orange line). The observed gain is in good agreement with the loss computed using the antenna patterns provided by the manufacturer and the azimuth shift determined using intensity correlation of $\mathrm{HH}$ and $\mathrm{VV}$ point target responses.

In Figure 9, the result of the above experiment is shown as the oversampled, coregistered azimuth response in the HV channel.

\section{Removal of Topographic Phase}

For the analysis of the topographic phase contribution removal, as described subsection II-E, the copolar (HH-VV) phase is chosen because it is the covariance element affected by an interferometric baseline that shows the highest overall coherence, making the effect of the baseline easier to visualize. An excerpt of the copolar phase difference is visualized in Figure 10 in radar coordinates; the phase assigned to the hue, the intensity to the corresponding $\mathbf{C}$ matrix element magnitude and by setting the saturation of the image with a sigmoid transformation of the copolar coherence magnitude, estimated using a $5 \times 2$ window.

\section{E. Polarimetric Calibration}

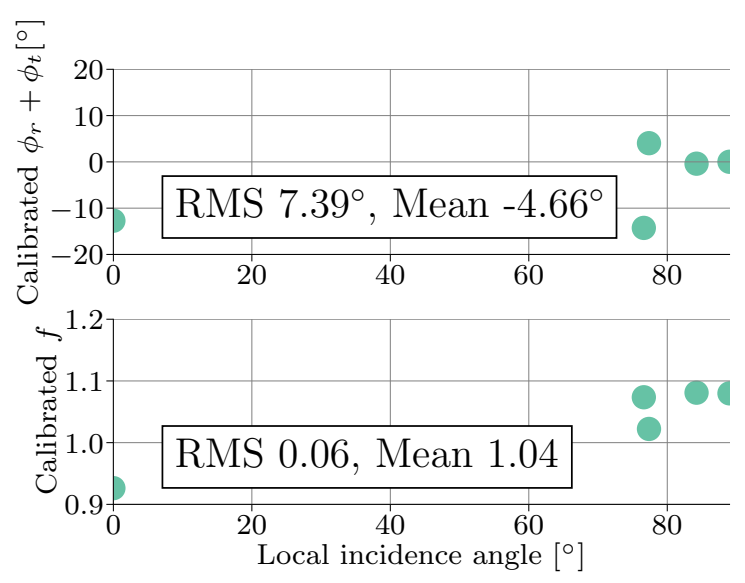

Fig. 11: Dependence of the residual copolar phase $\left(\phi_{r}+\right.$ $\phi_{t}$ ) and amplitude $(f)$ imbalances on the local incidence angle. The mean and RMS imbalances are shown in each plot. The reflector used for the determination of calibration parameters has been excluded from the plot.

\begin{tabular}{lcccc}
\hline Name & $\begin{array}{c}R_{0}[m] \\
\text { Range distance }\end{array}$ & $f$ & $\phi_{r}+\phi_{t}\left[^{\circ}\right]$ & Purity $[d B]$ \\
\hline CR1 & 74.3 & 0.93 & -12.71 & 39.3 \\
CR2 & 673.1 & 1.08 & -0.39 & 43.1 \\
CR3 & 824.6 & 1.08 & 0.02 & 45.1 \\
CR5 & 1,049 & 1.02 & 4.04 & 39.5 \\
CR6 & $2,689.3$ & 1.07 & -14.27 & 35.5 \\
\hline
\end{tabular}

TABLE V: Copolar phase $\left(\phi_{r}+\phi_{t}\right)$, amplitude imbalance $(f)$ and polarization purity (VV/HV ratio) computed on the reflectors using the calibrated dataset. The calibration reflector has been excluded from the table.

The methods described in the preceding sections were applied to prepare SLC images for each channel. For the final polarimetric calibration the procedure of subsection II-F was used; one reflector in the scene was used as a calibration target, with the four remaining reflectors used for the assessment of the calibration performance. An initial assessment of the data quality is made by computing polarization signatures [64] for two corner reflector located at different ranges. They are plotted in Figure 12. A quantitative evaluation of the calibration is obtained by estimating the residual copolar phase and amplitude imbalances $f$ and $\phi_{r}+\phi_{t}$ on the trihedrals after the calibration. The results are shown in Table V. In Figure 11, the dependence of the residuals on the local incidence angle is plotted; the angle was estimated using a $2 \mathrm{~m}$ posting digital elevation model of the scene that 
was backwards geocoded in the radar geometry.

For visual representation of the calibration results, the $\mathrm{HH}-\mathrm{VV}$ phase difference in radar coordinates is shown as a color image in Figure 10c. 


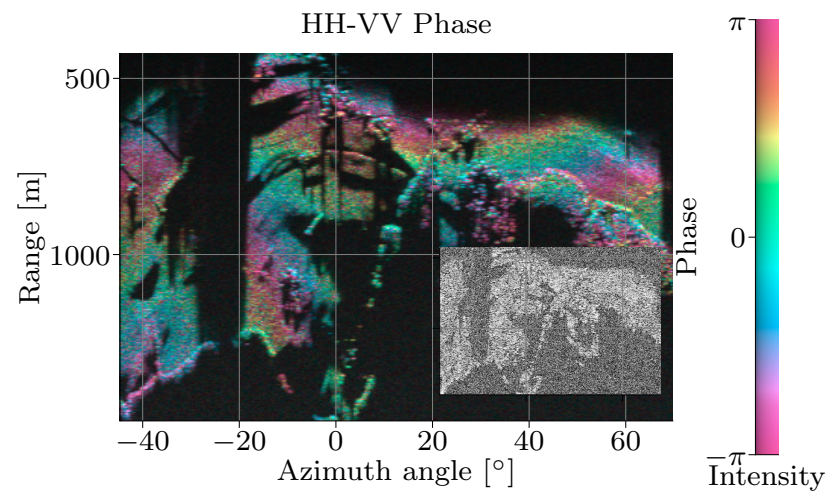

(a)

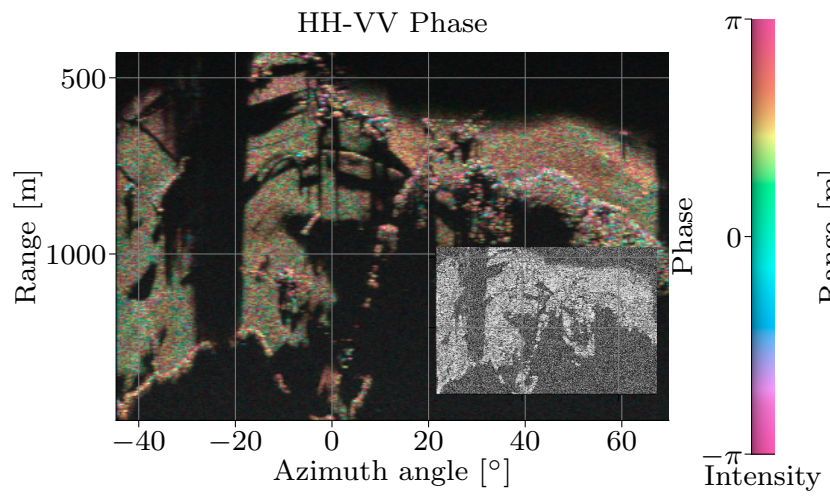

(b)

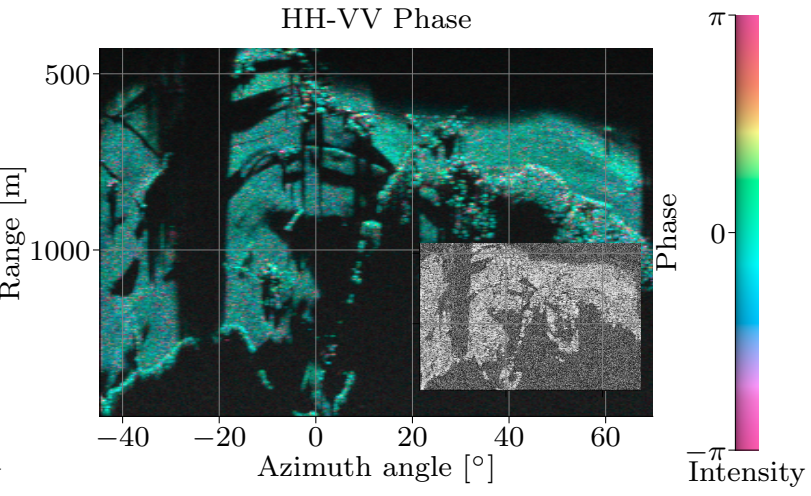

(c)

Fig. 10: HH-VV phase difference in radar coordinates, (a) before and (b) after the removal of the topographic phase term as described in subsection II-E; (c) calibrated copolar phase difference. The hue of the image is modulated by the covariance phase, the intensity by the magnitude, the saturation by the copolar coherence magnitude. The inset on the bottom left shows the copolar coherence magnitude. The interferometric fringe pattern visible in (a) is removed by the proposed correction, as plotted in (b). 
(a)

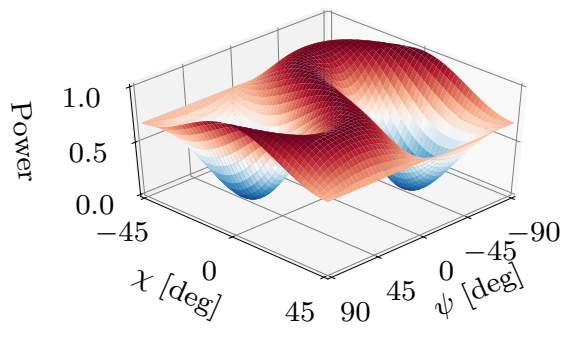

(c)

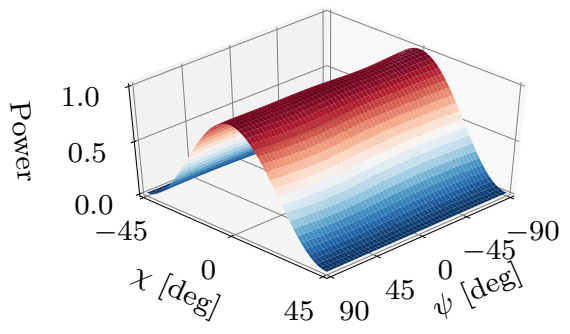

(a)

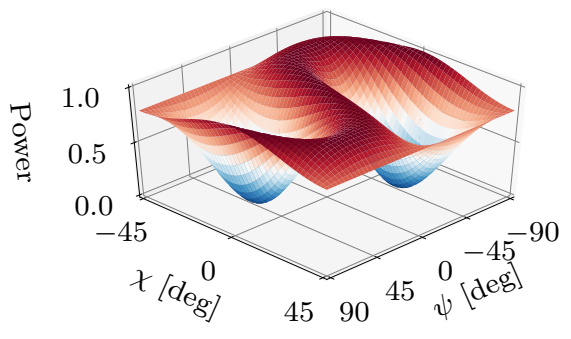

(c)

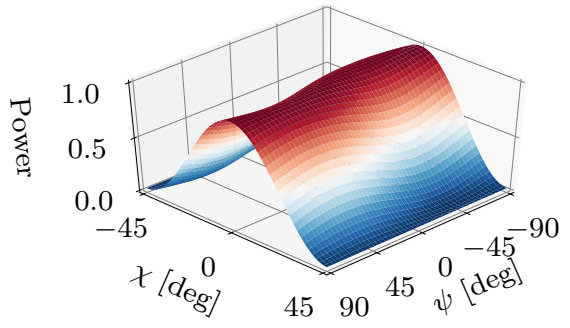

\section{CR2}

(b)

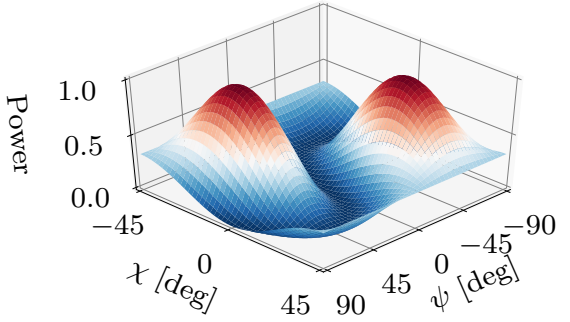

(d)

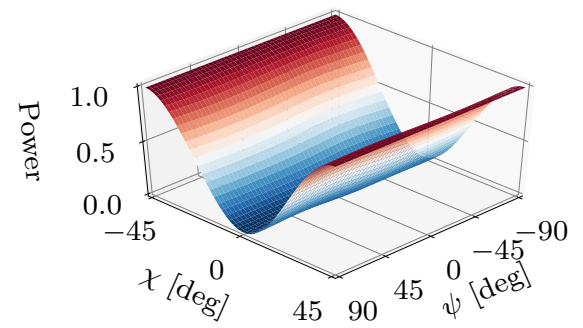

CR6

(b)

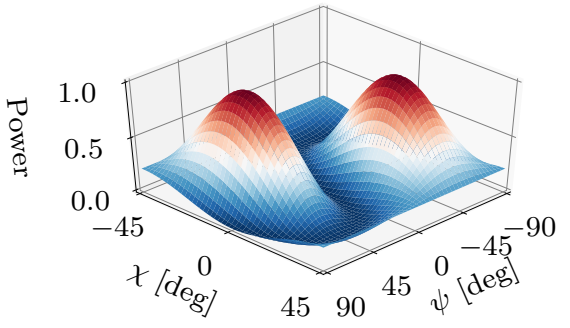

(d)

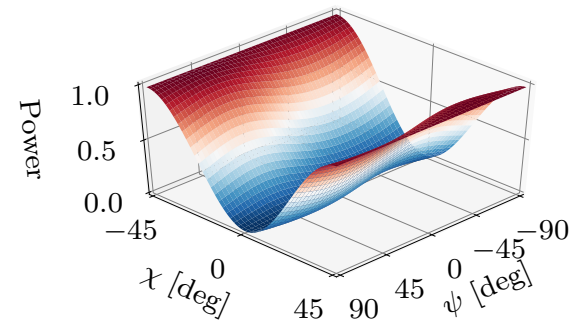

Fig. 12: Polarization signatures for two trihedral corner reflectors at the locations (a) "CR2" and (b) "CR6" . For both plots, each panel shows: (a) uncalibrated copolar signature, (b) uncalibrated crosspolar signature; (c) calibrated copolar signature,(d): calibrated crosspolar signature. The power of each response is normalized to the corresponding maximum. A distinct change in signature is observed after the calibration; it is mostly due to the removal of the HH-VV phase offset. 


\section{DISCUSSION}

\section{A. Beam Squint Correction}

The effect of the frequency squint on the raw data around TCR "CR2" is visible in Figure 6, panels (a) and (c) for the $\mathrm{HH}$ and $\mathrm{VV}$ channels. As described in subsection II-B and sketched in Figure 2, the data matrix appears skewed: the physical antenna direction and the effective pointing angle of the beam pattern only match for a brief time during each chirp due to the frequency scanning of the antenna. Because of that, if the data is range compressed, only part of the chirp bandwidth illuminates the target at each time, reducing the observed range resolution. This is verified in Figure 7 subfigures (a) and (c), where the oversampled response of TCR "CR2" after range compression is shown.

The linear squint factor $a$ estimated on all reflectors of the calibration array is given in Table III; the average estimated values of 4 and $3.9 \frac{\circ}{G H z}$ fit well with the figures suggested by the antenna manufacturer: 4.2 and $3.9 \frac{\circ}{G H z}$ for the $\mathrm{H}$ and $\mathrm{V}$ antennas respectively.

Thanks to the oversampled acquisition it is possible to use the proposed interpolation method to realign the samples in azimuth, compensating the effect of the squint by combining subsequent sub-chirps with different squint angles in a single coherent chirp that covers the entire bandwidth for the whole duration of time when the target is within the antenna beamwidth. This is shown in Figure 6 panels (b) and (d). The result of the interpolation is visible in Figure 6, panels (c) and (d): the spectrum is now aligned in azimuth; as a consequence the range resolution is decreased, as visible in Figure 7. Additionally the phase response seems to become more stable. Visually, the phase pattern observed in Figure 7 (a) and (c) is also removed entirely; however, a residual azimuthal phase can be observed in the $\mathrm{VV}$ channel in (e).

\section{B. Azimuth Processing}

The residual phase ramp observed on the "CR2" reflector in subsection IV-A is not unique to that object: A linear variation of 30 degrees over the $3 \mathrm{~dB}$ antenna beamwidth can be observed for all reflectors in the dataset, as plotted in Figure 8a. The model of subsection II-C was developed to explain this variation in terms of the acquisition geometry. The estimated phase center location values $\hat{L}_{p h}$ of Table IV are consistent and display a standard deviation of less than $2 \%$ of the antenna length, suggesting that the model is able to predict most of the phase variation. The estimated phase center shift for the $\mathrm{H}$ unit $\hat{L}_{p h}^{H}$ is less than $5 \mathrm{~cm}$, wile the one for the $\mathrm{V}$ antenna, $\hat{L}_{p h}^{V}$, is $-12 \mathrm{~cm}$, almost twice as large and with opposite sign. This difference presumably explains the steeper phase ramp in the VV channel: the range of $\theta_{r}$ for a scatterer is limited by the narrow antenna beamwidth $\theta_{3 d B}$. This should not cause a large phase variation, because evaluating (7) around zero should result in small values. However, considering the effect of $L_{p h}$, the entire function of Equation 12 is shifted by $\alpha$, simulating the effect of a larger $\theta_{r}$, as it would be obtained with a much bigger antenna beamwidth.

As shown in panel (g) of Figure 7, the azimuth phase variation is significantly reduced by the proposed correction method and the azimuth resolution is slightly degraded, although it is still better than $0.6^{\circ}$, the value that is expected by an incoherent average of azimuth samples. This result hints again that most of the phase ramp has been removed by the proposed method.

Similar results are observed for all trihedral reflectors, summarized in Figure 8b. The uncorrected HH channel shows a smaller variation, while the VV phase changes by $30^{\circ}$ over the antenna beamwidth. The observed phase slopes for the $\mathrm{V}$ and $\mathrm{H}$ antennas have opposite signs, as it is expected from the estimated phase center locations of Table IV.

After the correction, only the reflector "CR1" shows an appreciable residual azimuth phase variation; which is likely explained by its proximity to the radar relative to the antennas far field transition distance, in the order of $500 \mathrm{~m}$. A very similar variation is also observed in the HH channel. Excluding this exception, the residual phase inside the antenna beamwidth is under $5^{\circ}$.

\section{Antenna Pattern Misalignment}

Correcting for the estimated pattern alignment results in a gain of approx. $2.5 \mathrm{~dB}$ in $\mathrm{HV}$ power with respect to the uncompensated reference case, as plotted in Figure 9. The estimated gain is very close to the one expected by analyzing the patterns provided by the antenna manufacturer. This results confirms that the $1.8 \mathrm{~mm}$ shift setting employed to acquire the calibration data can correctly compensate the crosspolar power loss and the HH-VV misregistration.

\section{Removal of Topographic Phase}

The effect of the topographic phase compensation as described in subsection II-E is displayed in Figure 10; three topographic fringes are counted in the unflattened interferogram (Figure 10a), corresponding to a total phase variation of $9 \pi$. An estimate of this contribution is obtained from the unwrapped and rescaled interferogram 
between the upper and the lower HH channels. Because they are separated by a spatial baseline and they employ the same polarization (as seen in Figure 4), this interferogram provides an estimate of the topographic phase without additional polarimetric phase differences. The estimated topographic phase is then subtracted from the covariance matrix; the resulting flattened copolar phase difference is displayed in Figure 10b; no interferometric fringes can be counted. A more quantitative evaluation of the flattening process is obtained computing the correlation coefficient of the copolar phase and $\sin \left(\theta_{l}\right)$, where $\theta_{l}$ is the look vector elevation angle obtained from a digital elevation model (DEM). The estimated value is very close to zero, suggesting the correct removal of the topographic phase contribution:

if there would be a linear relationship between these two quantities, as expected from the approximate expression for the topographic phase of (17), a significant level of correlation would be observed. This measure cannot exclude residual nonlinear relationships between topography and the copolar phase; an additional visual verification was obtained by plotting their joint histogram; this did not display any discernible functional relationship. Therefore, it can be safely assumed that the flattening process is able to remove the topographic phase contribution. An additional advantage of this correction is the increased copolar coherence magnitude, as it can be by the inset coherence plot shown in Figure 10.

\section{E. Polarimetric Calibration}

An illustration of the effect of the calibration procedure of subsection II-F is given in Figure 12 by the polarization signatures of two reflectors before and after the calibration. The signature of the uncorrected data is distorted, the large variation of the maximum w.r.t the orientation angle is reminiscent of the response of a dihedral scatterer, which would show two distinct peaks. After calibration, both signatures show a fair correspondence with the expected polarization signature for trihedral reflectors. The dihedral-like response before the calibration is due to the $\mathrm{HH}-\mathrm{VV}$ phase imbalance, which shows a significant offset after the removal of the topographic contribution, as it can be seen in Figure 10b. By using a trihedral corner reflector or a similar oddbounce scatterer, this offset is estimated and removed. The effect of copolar phase imbalance correction is shown visually in Figure 10c; low entropy odd bounce scattering seems to be dominant in this part of the scene, which is mainly composed of open fields and wooded areas.

Amplitude and phase imbalances are successfully cor- rected by the proposed method; the mean residual for the amplitude imbalance $f$ is 1.03 with a root mean square deviation of 0.05 . A mean of $-4.5^{\circ}$ is observed for the copolar phase imbalance $\phi_{r}+\phi_{t}$, with a root mean square deviation of $7^{\circ}$. Two outliers, "CR1" and "CR6" heavily skew the estimated phase statistics; as shown in Figure 11. For "CR1", its placement on the ground together with its closeness to the radar could explain the offset in the copolar phase. The other extreme case is "CR6", that was placed at a large distance from the radar, this could result in a reduced polarization purity due to the larger influence of clutter in the larger resolution cell. Despite the outliers, no dependence of the residuals with the local incidence angle can be observed. This also suggests the validity of the method proposed in subsection II-F for the estimation and removal of the topographic contribution from the polarimetric phase differences.

Finally, the assumption of negligible crosstalk for the calibration model seems plausible considering that almost all reflectors exhibit polarization purities above 35 $\mathrm{dB}$.

\section{Conclusions}

In this paper, two main aspects of the calibration of KAPRI, a new polarimetric portable ground-based FMCW radar were discussed:

1) The preprocessing of raw data into SLC images, taking into account several effects due to the specific hardware design of the system.

2) The polarimetric calibration of data into phase and amplitude calibrated polarimetric covariance matrices.

\section{A. Preprocessing}

The particular antenna design causes a frequencydepending shift of the antenna mainlobe during the chirp that causes a worsened range resolution. It is corrected using a slow time-fast time interpolation procedure; significant range resolution improvement are observed after the correction.

The real aperture, azimuth scanning design results in a motion of the antenna phase center relative to the scatterers, causing an observable azimuth phase ramp in point target responses. The variation is significantly different between the antennas, with almost $30^{\circ}$ over the $3 \mathrm{~dB}$ beamwidth for the $\mathrm{V}$ antenna and much smaller for the $\mathrm{H}$ unit. This additional phase will complicate polarimetric calibration if left unaltered; this phase ramp is corrected by a SAR-like azimuth filter that reduces the total phase variation to under $10^{\circ}$. 
Because separated transmitting and receiving antennas are used for each polarization, the polarimetric calibration is more intricate due to the presence of an interferometric baseline between channels that adds a topographic phase contribution in the polarimetric phase differences. Using the cross-track interferometric baselines of KAPRI, the topographic contribution can be estimated and subtracted from each element of the covariance matrix affected by it.

\section{B. Calibration}

The resulting flattened covariance matrix is then calibrated by assuming zero crosstalk and estimating copolar imbalances using a trihedral corner reflector assuming the parameters to be independent from the incidence angle. The crosspolar imbalance is estimated using distributed targets under the assumption of reciprocity.

The calibration quality is assessed by estimating residual calibration parameter on a calibrated scene with five trihedral corner reflectors: the mean amplitude imbalance is close to unity while the mean residual phase imbalance is very close to zero, with an RMS of $7^{\circ}$; no significant variation with incidence angle is observed. These results suggest that the simplified calibration model [62], [63] is suitable to calibrate fully polarimetric KAPRI data.

\section{REFERENCES}

[1] A. K. Gabriel, R. M. Goldstein, and H. A. Zebker, "Mapping small elevation changes over large areas - Differential radar interferometry," Journal of Geophysical Research, vol. 94, no. B7, pp. 9183-9191, 1989.

[2] D. Massonnet and T. Rabaute, "Radar interferometry: Limits and potential," IEEE Transactions on Geoscience and Remote Sensing, vol. 31, no. 2, pp. 455-464, mar 1993.

[3] P. Rosen, S. Hensley, I. Joughin, F. Li, S. Madsen, E. Rodriguez, and R. Goldstein, "Synthetic aperture radar interferometry," Proceedings of the IEEE, vol. 88, no. 3, pp. 333-382, mar 2000.

[4] R. Bamler and P. Hartl, "Synthetic aperture radar interferometry," Inverse Problems, vol. 14, pp. R1-R54, 1999.

[5] D. L. Galloway, K. W. Hudnut, S. E. Ingebritsen, S. P. Phillips, G. Peltzer, F. Rogez, and P. a. Rosen, "Detection of aquifer system compaction and land subsidence using interferometric synthetic aperture radar, Antelope Valley, Mojave Desert, California," Water Resources Research, vol. 34, no. 10, p. 2573, oct 1998.

[6] T. Strozzi, U. Wegmüller, L. Tosi, G. Bitelli, and V. Spreckels, "Land subsidence monitoring with differential SAR interferometry," Photogrammetric Engineering \& Remote Sensing, vol. 67, no. 11 , pp. 1261-1270, 2001.

[7] D. L. Galloway and J. Hoffmann, "The application of satellite differential SAR interferometry-derived ground displacements in hydrogeology," Hydrogeology Journal, vol. 15, no. 1, pp. 133154, 2007.

[8] D. Massonnet, P. Briole, and A. Arnaud, "Deflation of Mount Etna monitored by spaceborne radar interferometry," Nature, vol. 375, no. 6532, pp. 567-570, jun 1995.
[9] R. M. Goldstein, H. Engelhardt, B. Kamb, and R. M. Frolich, "Satellite radar interferometry for monitoring ice sheet motion: application to an antarctic ice stream." Science, vol. 262, no. 5139, pp. 1525-1530, 1993.

[10] J. J. Mohr, N. Reeh, and S. N. Madsen, "Three-dimensional glacial flow and surface elevation measured with radar interferometry," Nature, vol. 391, pp. 273-276, 1998.

[11] C. Carnec, D. Massonnet, and C. King, "Two examples of the use of SAR interferometry on displacement fields of small spatial extent," Geophysical Research Letters, vol. 23, no. 24, p. 3579, 1996.

[12] F. Catani, P. Farina, S. Moretti, G. Nico, and T. Strozzi, "On the application of SAR interferometry to geomorphological studies: Estimation of landform attributes and mass movements," Geomorphology, vol. 66, no. 1-4 SPEC. ISS., pp. 119-131, 2005.

[13] D. Massonnet, M. Rossi, C. Carmona, F. Adragna, G. Peltzer, K. Feigl, and T. Rabaute, "The displacement field of the Landers earthquake mapped by radar interferometry," Nature, vol. 364, no. 6433, pp. 138-142, jul 1993.

[14] H. A. Zebker, P. A. Rosen, R. M. Goldstein, A. Gabriel, and C. L. Werner, "On the derivation of coseismic displacement fields using differential radar interferometry: The Landers earthquake," Journal of Geophysical Research, vol. 99, no. B10, pp. 1961719634, 1994.

[15] S. R. Cloude and E. Pottier, "An entropy based classification scheme for land applications of polarimetric SAR," IEEE Transactions on Geoscience and Remote Sensing, vol. 35, no. 1, pp. 68-78, 1997.

[16] J.-S. Lee, M. Grunes, T. L. Ainsworth, L.-J. Du, D. Schuler, and S. Cloude, "Unsupervised classification using polarimetric decomposition and the complex Wishart classifier," IEEE Transactions on Geoscience and Remote Sensing, vol. 37, no. 5, pp. 2249-2258, sep 1999.

[17] I. Hajnsek, E. Pottier, and S. R. Cloude, "Inversion of surface parameters from polarimetric SAR," IEEE Transactions on Geoscience and Remote Sensing, vol. 41, no. 4, pp. 727-744, 2003.

[18] F. T. Ulaby, D. Held, M. C. Donson, K. C. McDonald, and T. B. A. Senior, "Relating polarization phase difference of SAR signals to scene properties," IEEE Transactions on Geoscience and Remote Sensing, vol. GE-25, no. 1, pp. 83-92, jan 1987.

[19] S. Leinss, G. Parrella, and I. Hajnsek, "Snow height determination by polarimetric phase differences in X-Band SAR data," IEEE Journal of Selected Topics in Applied Earth Observations and Remote Sensing, vol. 7, no. 9, pp. 3794-3810, sep 2014.

[20] L. Pipia, X. Fabregas, A. Aguasca, C. Lopez-Martinez, S. Duque, J. Mallorqui, and J. Marturia, "Polarimetric differential SAR interferometry: first results with ground-based measurements," IEEE Geoscience and Remote Sensing Letters, vol. 6, no. 1, pp. 167-171, jan 2009.

[21] R. Iglesias, D. Monells, X. Fabregas, J. J. Mallorqui, A. Aguasca, and C. Lopez-Martinez, "Phase quality optimization in polarimetric differential SAR interferometry," IEEE Transactions on Geoscience and Remote Sensing, vol. 52, no. 5, pp. 2875-2888, 2014.

[22] D. Leva, G. Nico, D. Tarchi, and J. Fortuny-Guasch, "Temporal analysis of a landslide by means of a ground-based SAR interferometer," IEEE Transactions on Geoscience and Remote Sensing, vol. 41, no. 4 PART I, pp. 745-752, 2003.

[23] H. Rudolf, D. Leva, D. Tarchi, and A. Sieber, "A mobile and versatile SAR system," in Proceedings of the IEEE International Geoscience and Remote Sensing Symposium, vol. 1, no. 2, 1999, pp. 592-594.

[24] M.-K. Kang, K.-E. Kim, H. Lee, S.-J. Cho, and J.-H. Lee, "Preliminary results of polarimetric characteristics for C-band quad-polarization GB-SAR images using H / A / a polarimetric decomposition theorem," Korean Journal of Remote Sensing, vol. 25, no. 6, pp. 531-546, 2009.

[25] A. Aguasca, A. Broquetas, J. J. Mallorqui, and X. Fabregas, "A 
solid state L to X-band flexible ground-based SAR system for continuous monitoring applications," in Proceedings of the IEEE International Geoscience and Remote Sensing Symposium, vol. 2, no. C, 2004, pp. 757-760.

[26] L. Pipia, X. Fabregas, A. Aguasca, C. Lopez-Martinez, J. J. Mallorqui, and O. Mora, "A subsidence monitoring project using a polarimetric GB-SAR sensor," European Space Agency, (Special Publication) ESA SP, no. SP-644, 2007.

[27] C. Werner, T. Strozzi, A. Wiesmann, and U. Wegmüller, "A real-aperture radar for ground-based differential interferometry," Proceedings of the IEEE International Geoscience and Remote Sensing Symposium, vol. 3, no. 1, pp. 210-213, jul 2008.

[28] S. Rödelsperger, A. Coccia, D. Vicente, and A. Meta, "Introduction to the new metasensing ground-based SAR: Technical description and data analysis," in Proceedings of the IEEE International Geoscience and Remote Sensing Symposium, 2012, pp. 4790-4792.

[29] C. Werner, A. Wiesmann, T. Strozzi, A. Kos, R. Caduff, and U. Wegmüller, "The GPRI multi-mode differential interferometric radar for ground-based observations," in Proceedings of the European Conference on Synthetic Aperture Radar. VDE, 2012, pp. 304-307.

[30] R. Caduff, F. Schlunegger, A. Kos, and A. Wiesmann, "A review of terrestrial radar interferometry for measuring surface change in the geosciences," Earth Surface Processes and Landforms, vol. 40, no. 2, pp. 208-228, feb 2015.

[31] O. Monserrat, M. Crosetto, and G. Luzi, "A review of groundbased SAR interferometry for deformation measurement," ISPRS Journal of Photogrammetry and Remote Sensing, vol. 93, no. 0, pp. 40-48, 2014.

[32] J. C. Bennett and K. Morrison, "Development of a ground-based, polarimetric synthetic aperture radar," Aerospace Applications Conference, 1996. Proceedings., 1996 IEEE, vol. 4, pp. 139-146 vol.4, 1996.

[33] J. C. Bennett, K. Morrison, A. M. Race, S. Quegan, H. Building, and H. Road, "The UK NERC fully portable polarimetric groundbased synthetic aperture radar (GB-SAR)," in Proceedings of the European Conference on Synthetic Aperture Radar, 2000, pp. 2313-2315.

[34] Z. S. Zhou, W. M. Boerner, and M. Sato, "Development of a ground-based polarimetric broadband SAR system for noninvasive ground-truth validation in vegetation monitoring," IEEE Transactions on Geoscience and Remote Sensing, vol. 42, no. 9, pp. 1803-1810, 2004.

[35] T. Hamasaki, M. Sato, L. Ferro-Famil, and E. Pottier, "Natural objects monitoring using polarimetric interferometric groundbased SAR (GB-SAR) system," in Proceedings of the IEEE International Geoscience and Remote Sensing Symposium, vol. 6 , no. 1. IEEE, 2005, pp. 4092-4095.

[36] R. Iglesias, A. Aguasca, X. Fabregas, J. J. Mallorqui, D. Monells, C. Lopez-Martinez, and L. Pipia, "Ground-based polarimetric SAR interferometry for the monitoring of terrain displacement phenomena-part I: theoretical description," IEEE Journal of Selected Topics in Applied Earth Observations and Remote Sensing, vol. 8, no. 3, pp. 1-1, 2014.

[37] L. Pipia, "Polarimetric Differential SAR Interferometry With Ground-Based Sensors," Ph.D. dissertation, Universitat Politècnica de Catalunya, 2009.

[38] L. Pipia, X. Fabregas, A. Aguasca, and C. Lopez-Martinez, "Polarimetric temporal analysis of urban environments with a ground-based SAR," IEEE Transactions on Geoscience and Remote Sensing, vol. 51, no. 4, pp. 2343-2360, 2013.

[39] M.-K. Kang, K.-E. Kim, H. Lee, S.-J. Cho, and J.-H. Lee, "Preliminary result of polarization property analysis using fully polarimetric GB-SAR images," in Proceedings of the IEEE International Geoscience and Remote Sensing Symposium. IEEE, jul 2010, pp. 4019-4022.

[40] T. G. Yitayew, "High resolution three-dimensional imaging of sea ice using ground- based tomographic SAR data," in Proceedings of the European Conference on Synthetic Aperture Radar, 2014, pp. 2-5.

[41] O. Frey, C. L. Werner, and A. Wiesmann, "Tomographic profiling of the structure of a snow pack at X-/Ku-Band using SnowScat in SAR mode," in Proceedings of the European Radar Conference, vol. 1. IEEE, sep 2015, pp. 1-4.

[42] O. Frey, C. L. Werner, R. Caduff, and A. Wiesmann, "A time series of SAR tomographic profiles of a snowpack," Proceedings of the European Conference on Synthetic Aperture Radar, pp. 726-730, 2016

[43] S. S. Cherukumilli, "GBIR crosstalk reduction of fully polarimetric data from Blue Springs Dam," Master Thesis, University of Missouri - Columbia, 2012.

[44] S. Baffelli, O. Frey, and I. Hajnsek, "System Characterization and Polarimetric Calibration of the Ku- Band Advanced Polarimetric Interferometer," in Proceedings of the European Conference on Synthetic Aperture Radar, 2016, pp. 2-5.

[45] T. Strozzi, C. Werner, A. Wiesmann, and U. Wegmüller, “Topography mapping with a portable real-aperture radar interferometer," IEEE Geoscience and Remote Sensing Letters, vol. 9, no. 2, pp. 277-281, mar 2011.

[46] A. Stove, "Linear FMCW radar techniques," IEE Proceedings F Radar and Signal Processing, vol. 139, no. 5, p. 343, oct 1992.

[47] J. N. Hines, V. H. Rumsey, and C. H. Walter, "Traveling-Wave Slot Antennas," Proceedings of the IRE, vol. 41, no. 11, pp. 16241631, 1953.

[48] C. Granet, G. L. James, and A. Ross Forsyth, "Aperture Antennas: Waveguides and Horns," in Modern Antenna Handbook, Constantine A. Balanis, Ed. John Wiley \& Sons, Ltd, 2007, pp. 97-156.

[49] R. K. Enjiu and M. B. Perotoni, "Slotted waveguide antenna design using 3D EM simulation," Microwave Journal, no. July, 2013.

[50] S.-T. Yang and H. Ling, "Combining a frequency-scanned antenna and a short-pulse radar for 2-D imaging," in 2014 IEEE Antennas and Propagation Society International Symposium (APSURSI). IEEE, jul 2014, pp. 137-138.

[51] — , "Range-azimuth tracking of humans using a microstrip leaky wave antenna," in Proceedings of the 2012 IEEE International Symposium on Antennas and Propagation. IEEE, jul 2012, pp. 1-2.

[52] W. Mayer, M. Wetzel, and W. Menzel, "A novel direct-imaging radar sensor with frequency scanned antenna," in IEEE MTT-S International Microwave Symposium Digest, 2003, vol. 3. IEEE, 2003, pp. 1941-1944.

[53] Y. Alvarez, R. Camblor, C. Garcia, J. Laviada, C. Vazquez, S. Ver-Hoeye, G. Hotopan, M. Fernandez, A. Hadarig, A. Arboleya, and F. Las-Heras, "Submillimeter-Wave Frequency Scanning System for Imaging Applications," IEEE Transactions on Antennas and Propagation, vol. 61, no. 11, pp. 5689-5696, 2013.

[54] J. D. Kraus, Antennas, 2nd ed. McGraw-Hill, 1988.

[55] R. Raney, T. Freeman, R. Hawkins, and R. Bamler, "A plea for radar brightness," in Proceedings of the IEEE International Geoscience and Remote Sensing Symposium, vol. 2. IEEE, 1994, pp. 1090-1092.

[56] H. Lee, J.-H. Lee, K.-E. Kim, N.-H. Sung, and S.-J. Cho, "Development of a Truck-Mounted Arc-Scanning Synthetic Aperture Radar," IEEE Transactions on Geoscience and Remote Sensing, vol. 52, no. 5, pp. 2773-2779, may 2014.

[57] P. Beasley, A. Stove, B. Reits, and B. As, "Solving the problems of a single antenna frequency modulated CW radar," in IEEE International Conference on Radar. IEEE, 1990, pp. 391-395.

[58] D. Massonnet, H. Vadon, and M. Rossi, "Reduction of the need for phase unwrapping in radar interferometry," IEEE Transactions on Geoscience and Remote Sensing, vol. 34, no. 2, pp. 489-497, mar 1996. 
[59] K. Sarabandi, "A Convenient Technique For Polarimetric Calibration of Single-Antenna Radar Systems," IEEE Transactions on Geoscience and Remote Sensing, vol. 28, no. 6, pp. 1022-1033, 1990.

[60] K. Sarabandi, L. E. Pierce, and F. T. Ulaby, "Calibration of a polarimetric imaging SAR," IEEE Transactions on Geoscience and Remote Sensing, vol. 30, no. 3, pp. 540-549, may 1992.

[61] T. L. Ainsworth, L. Ferro-Famil, and Jong-Sen Lee, "Orientation angle preserving a posteriori polarimetric SAR calibration," IEEE Transactions on Geoscience and Remote Sensing, vol. 44, no. 4, pp. 994-1003, apr 2006.

[62] A. G. Fore, B. D. Chapman, B. P. Hawkins, S. Hensley, C. E. Jones, T. R. Michel, and R. J. Muellerschoen, "UAVSAR polarimetric calibration," IEEE Transactions on Geoscience and Remote Sensing, vol. 53, no. 6, pp. 3481-3491, jun 2015.

[63] K. Sarabandi, F. Ulaby, and M. Tassoudji, "Calibration of polarimetric radar systems with good polarization isolation," IEEE Transactions on Geoscience and Remote Sensing, vol. 28, no. 1, pp. 70-75, 1990 .

[64] J. J. van Zyl, H. A. Zebker, and C. Elachi, "Imaging radar polarization signatures: Theory and observation," Radio Science, vol. 22, no. 4, pp. 529-543, 1987.

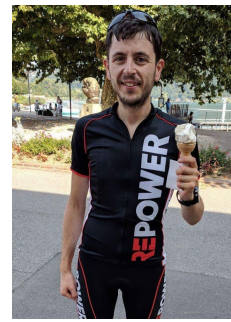

Simone Baffelli (S'13) Received the BSc. and MSc. titles in electrical engineering from the Swiss Federal Institute of Technology (ETH) in Zürich, Switzerland in 2011 and 2013 respectively. He is currently a doctoral student at the Chair of Earth Observation and Remote Sensing, Swiss Federal Institute of Technology (ETH) in Zürich, Switzerland. His current research interests include the use of ground based radar systems for the mapping of surface displacement, differential interferometry and the processing and calibration of ground based radar data.

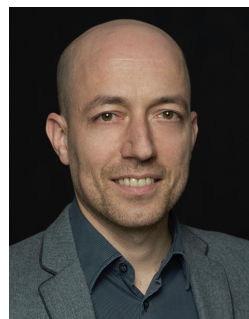

Othmar Frey (S'04-M'08-SM'17) received the M.Sc. degree in geomatic engineering (Hons.) from ETH Zurich, Zurich, Switzerland, in 2002, and the Ph.D. (Dr. sc. nat.) degree (Hons.) in radar remote sensing from the University of Zurich, in 2010. Othmar Frey is a tenured Senior Researcher and Lecturer at ETH Zurich, Zurich, Switzerland, and also a Senior Scientist at GAMMA Remote Sensing AG, Gümligen, Switzerland. He has been working in this joint academic/industry setting since 2011. Previously, from 2002 to 2010, he was a Research Associate with the Remote Sensing Laboratories, University of Zurich. His research encompasses 2-D and 3-D (tomographic) synthetic aperture radar (SAR) focusing techniques and interferometric techniques for applications such as 3-D forest mapping, ground deformation monitoring (persistent scatterer interferometry / differential tomography), and profiling the structure of snowpacks, using spaceborne, airborne, and terrestrial radar sensors. He has been active in various national and international research projects, as a PI and project manager as well as a co-investigator. At GAMMA he has also been active in the development of the Gamma Software and in technical consulting related to SAR imaging and interferometric applications. He has been a member of the SAOCOM-CS Science Expert Group with the European Space Agency. Since 2015, he has been the chair of the Swiss Chapter of the IEEE Geoscience and Remote Sensing Society. Dr. Frey received the distinction award and prize of the Faculty of Science, University of Zurich, for his Ph.D. thesis, in 2010, and he received the ETH medal for an outstanding M.Sc. thesis, in 2002. He was the recipient of the 3rd Place Student Paper Award at the 2010 EUSAR Conference in Aachen, Germany, and he was a co-recipient of the Best Paper Award at the ISPRS Workshop Laserscanning and Silvilaser 2007 in Helsinki, Finland. 


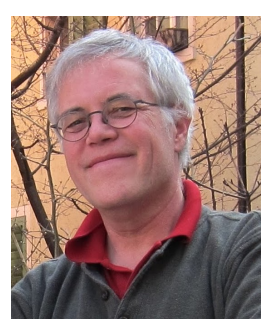

Charles Werner (S'75-M'78-SM'08) Dr. Charles Werner attended the University of Pennsylvania where he received a MS degree in Electrical Engineering (1981) and $\mathrm{PhD}$ degree in Systems Engineering (1987). During his studies at Penn, he developed a polarimetric 3-D microwave imaging facility covering 2-18 $\mathrm{GHz}$ and performed research on 3-D imaging of incoherent acoustic and microwave noise sources. At NASA JPL in Pasadena, California (1982-1999), he worked on applications of airborne and space borne SAR . This included focusing algorithms, speckle reduction, scatterer classification, polarization signatures, detection of moving targets and absolute and relative radiometric calibration of polarimetric SAR data. He developed the system design and performed system analysis for the Cassini RADAR instrument, a multi-mode radar incorporating a SAR, altimeter, scatterometer and radiometer for mapping Saturn's largest moon, Titan Maps produced by this radar reveal the existence of hydrocarbon lakes and rivers, mountains, and ice sand dunes on Titan's surface. While working at the University of Zurich (1992-95), he developed an endto-end interferometric SAR processing system for both airborne and space borne sensors included ERS and the Dornier DOSAR airborne radar. He was responsible for the implementation and testing of the interferometric phase unwrapping algorithms used in the Shuttle Radar Topographic Mission (SRTM) Topographic Processing System (TPS) and the GeoSAR P- and X- Band airborne SAR systems. In 1995 , together with Urs Wegmuller, he founded Gamma Remote Sensing AG, located in Gumligen, Switzerland, to promote radar remote sensing, particularly SAR interferometry, for a wide range of applications including generation of digital elevation models, mapping interferometric signatures of forest and agricultural areas, and measurement of crustal deformation, landslides, and ice motion with DINSAR. He is the principle developer of the Gamma radar software that includes generation and advanced filtering of interferometric and intensity radar images, terrain geocoding, time-series analysis, and visualization tools. He developed the Snowscat polarimetric 9-18 GHz scatterometer and ELBARA-II and ELBARA-III L-band radiometers for ESA. He is the developer of the Gamma Terrestrial Radar Interferometer (GPRI2), a ground-based $\mathrm{Ku}-\mathrm{Band} \mathrm{FM}-\mathrm{CW}$ radar interferometer for producing insitu deformation maps and digital elevation models useful for glacier mapping, and monitoring slope stability and infrastructure. Currently, he is developing a polarimetric wideband scatterometer (WBSCAT) covering 1-40 GHz for ESA, and a compact terrestrial L-Band SAR.

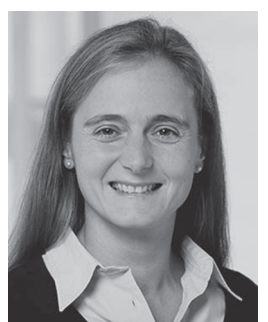

Irena Hajnsek (A'01 - M'06 - SM'09 F'14) received the Dipl. Degree (Honors) from the Free University of Berlin, Berlin, Germany, in 1996 and the Dr. degree (Honors) from the Friedrich Schiller University Jena, Jena, Germany, in 2001. Since November 2009, she has been a Professor of Earth observation with the Institute of Environmental Engineering, Swiss Federal Institute of Technology (ETH) Zurich, Zurich, Switzerland, and, at the same time, the Head of the Polarimetric SAR Interferometry Group, Microwaves and Radar Institute (HR), German Aerospace Center (DLR), Wessling, Germany. From 1996 to 1999, she was with the DLR-HR. From 1999 to 2000, she was with the Institut d'Electronique et de Telecommunications de Rennes, University of Rennes 1, Rennes, France for ten months, in the frame of the EC-TMR Radar Polarimetry Network. In 2005, she was a Guest Scientist with the University of Adelaide, Adelaide, Australia, for six weeks. She is the Science Coordinator of the German satellite mission TanDEM-X. Her main research interests are in electromagnetic propagation and scattering theory, radar polarimetry, SAR and interferometric SAR data processing techniques, and environmental parameter modelling and estimation. Dr. Hajnsek was a member of the ESA Mission Advisory Group of the $7^{\text {th }}$ Explorer Mission CoReH2O from 2009 to 2013. She was a Technical Program Cochair of the 2012 IEEE International Geoscience and Remote Sensing Symposium in Munich. Since 2013, she has been a member of the IEEE Geoscience and Remote Sensing Society Administrative Committee. 